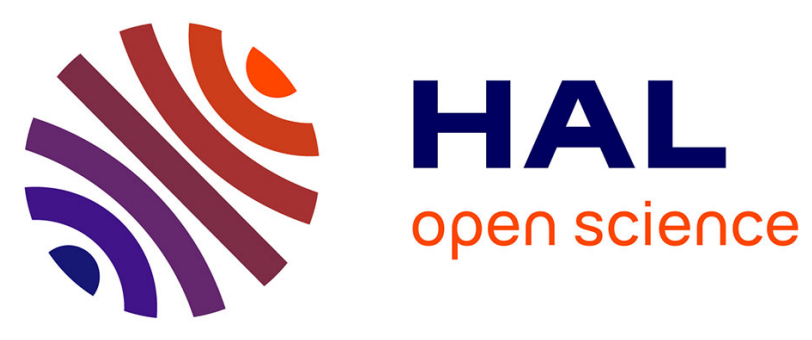

\title{
Two field multibody method for periodic homogenization in fracture mechanics of nonlinear heterogeneous materials
}

Frédéric Perales, Stéphane Bourgeois, André Chrysochoos, Yann Monerie

\section{- To cite this version:}

Frédéric Perales, Stéphane Bourgeois, André Chrysochoos, Yann Monerie. Two field multibody method for periodic homogenization in fracture mechanics of nonlinear heterogeneous materials. Engineering Fracture Mechanics, 2008, Local Approach to Fracture (1986-2006): Selected papers from the 9th European Mechanics of Materials Conference, 75 (11), pp.3378-3398. 10.1016/j.engfracmech.2007.07.017 . hal-00572322

\section{HAL Id: hal-00572322 \\ https://hal.science/hal-00572322}

Submitted on 29 Nov 2016

HAL is a multi-disciplinary open access archive for the deposit and dissemination of scientific research documents, whether they are published or not. The documents may come from teaching and research institutions in France or abroad, or from public or private research centers.
L'archive ouverte pluridisciplinaire HAL, est destinée au dépôt et à la diffusion de documents scientifiques de niveau recherche, publiés ou non, émanant des établissements d'enseignement et de recherche français ou étrangers, des laboratoires publics ou privés. 


\title{
Two field multibody method for periodic homogenization in fracture mechanics of nonlinear heterogeneous materials
}

\author{
F. Perales ${ }^{\mathrm{a}, *}$, S. Bourgeois ${ }^{\mathrm{c}}$, A. Chrysochoos ${ }^{\mathrm{b}}, \mathrm{Y}$. Monerie ${ }^{\mathrm{a}}$ \\ a Institut de Radioprotection et de Sûreté Nucléaire, DPAM, SEMCA, LEC, BP3, 13115 St Paul Lez Durance cedex, France \\ b Laboratoire de Mécanique et Génie Civil, CC048, Place E. Bataillon, 34095 Montpellier cedex 5, France \\ ' Centrale Marseille \& LMA (CNRS-UPR7051), Technopôle de Château-Gombert, 38, rue Joliot Curie, 13451 Marseille cedex 20, France
}

\begin{abstract}
This paper presents a new computational approach dedicated to the fracture of nonlinear heterogeneous materials. This approach extends the standard periodic homogenization problem to a two field cohesive-volumetric finite element scheme. This two field finite element formulation is written as a generalization Non-Smooth Contact Dynamics framework involving Frictional Cohesive Zone Models. The associated numerical platform allows to simulate, at finite strain, the fracture of nonlinear composites from crack initiation to post-fracture behavior. The ability of this computational approach is illustrated by the fracture of the hydrided Zircaloy under transient loading.
\end{abstract}

Keywords: Periodic homogenization; Frictional cohesive zone model; Dynamic fracture; Zircaloy-4

\section{Introduction}

The dynamic fracture of heterogeneous materials (composites, Functionally Graded Materials, porous materials) is a challenging problem in many engineering fields [1]. Due to the presence of heterogeneities, the pertinent scale to analyse the ultimate overall properties of such materials, as the effective dynamic fracture toughness, is the microscopic scale. At this scale, various mechanisms of nonlinear damage have to be invoked: void growth and coalescence, crack initiation and branching, mixed mode crack growth at the interface between the different phases, post-fracture frictional contact on the crack lips. A convenient numerical modeling of the dynamic fracture of the heterogeneous materials has thus to be able to deal with nonlinear and non-smooth volumetric and surface behaviors at the microscale.

In the frame of the French "Institut de Radioprotection et de Sûreté Nucléaire" (IRSN) research program on nuclear fuel safety under accident conditions, a new computational micromechanical approach has been developed to analyse the effects of the microstructure heterogeneity on the overall material behavior. The 
micromechanical approach rests on an extension of the standard periodic homogenization based both on the concept of Frictional Cohesive Zone Model (FCZM) and on a multibody method in the context of the NonSmooth Contact Dynamics (NSCD) [2,3].

The FCZMs allow the simulation of crack initiation, crack propagation and post-fracture non-smooth behavior on the crack lips such as frictional contact [4,5]. These models consist in a softening traction-separation relation on the crack lips within a multibody framework: each mesh is considered as an independent body connected to another by nonlinear and non-smooth relations [6,7]. Since velocity can become discontinuous during dynamic fracture [8], the NSCD approach $[9,10]$ is used to treat the post-fracture frictional contact without any regularization nor penalization.

The effective properties related to fracture mechanics are obtained by periodic numerical homogenization. The standard periodic problem $[11,12]$ is extended to the multibody approach. The Finite Element formulation becomes a two field Finite Element formulation, including a periodic displacement field and an average deformation gradient field. The NSCD framework is thus extended to these fields introducing a new discrete mapping.

The associated numerical platform is composed of three libraries with Object-Oriented Programming: a Fortran90 library dedicated to surface behaviors related to FCZM in the NSCD approach (LMGC90 [13]), a $\mathrm{C}++$ library dedicated to periodic Finite Element modeling (PELICANS [14]) and a C ++ library dedicated to bulk constitutive models (MAтLiв [15]). This software allows simulating the fracture of the heterogeneous materials at finite strain.

This paper focusses on the ability of the proposed two field multibody method dealing with the overall fracture properties of heterogeneous materials. The questions related to the cohesive-volumetric finite element scheme - in particular, to the difficulties often reported in the literature (mesh sensitivity, crack path, dependence of the crack speed to the loading rate, etc.) - are out of the scope of the present paper and are addressed in a forthcoming paper. However, some hints are given on the relative independence of the overall fracture properties. The ability of the software and of the proposed strategy is illustrated by the fracture of a heterogeneous material, the hydrided Zircaloy-4, under transient loading. The effective properties are determined on random periodic representative volume element.

\section{Fracture modeling}

\subsection{Multibody approach}

The dynamic fracture of heterogeneous materials is studied through a micromechanical modeling based on a multibody concept and FCZM. Each element of a Finite Element mesh is considered as an independent body connected to each other with mixed interface relationship (see Fig. 1). The overall progressive damageable behavior is thus obtained by coupling (see Fig. 2):

- The volumetric behavior inside the meshes, describing the behavior without any damage.

- The FCZM surface properties between the meshes, taking damage processes into account.

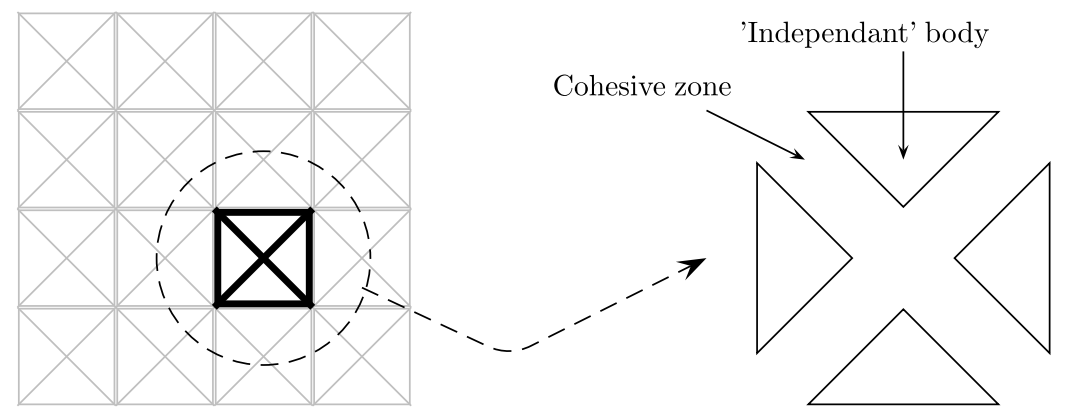

Fig. 1. Multibody strategy: each finite element is a body. 


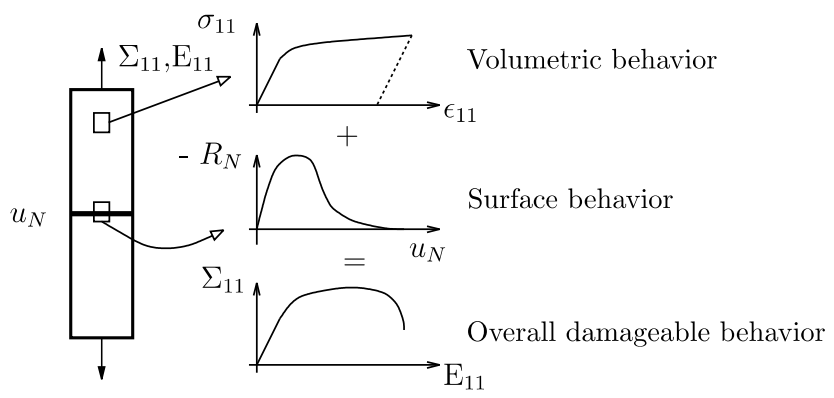

Fig. 2. Decoupling of volumetric behavior and surface behavior: the damageable behavior is described by FCZM.

\subsection{Frictional cohesive zone model}

The FCZM is a representation of the local physics of the fracture processes, from crack initiation to postfracture unilateral conditions, at the crack tip. The faces of a Finite Element mesh are linked up by softening traction-separation relations (see Fig. 1). The nonlinear softening relations considered in this study rests on the cohesive-friction coupling proposed by Raous et al. [4] and on an irreversible surface damage law. A cohesive stress, called $\mathbf{R}^{\text {coh }}$, is introduced in the Signorini-Coulomb problem 1,2 and related to the displacement jump $[\boldsymbol{u}]$ across the future crack lips (3):

$$
\begin{aligned}
& -\left(R_{\mathrm{N}}+R_{\mathrm{N}}^{\mathrm{coh}}\right) \in \partial I_{\mathbb{R}_{+}}\left(u_{\mathrm{N}}\right), \\
& \left(\mathbf{R}_{\mathrm{T}}+\mathbf{R}_{\mathrm{T}}^{\mathrm{coh}}\right) \in \partial_{\dot{\mathbf{u}}_{\mathrm{T}}}\left(\mu\left|R_{\mathrm{N}}+R_{\mathrm{N}}^{\mathrm{coh}}\right|\left\|\dot{\mathbf{u}}_{\mathrm{T}}\right\|\right), \\
& \left.\mathbf{R}^{\mathrm{coh}}=\beta \quad C_{\mathrm{N}} \boldsymbol{n} \otimes \boldsymbol{n}+C_{\mathrm{T}} \frac{\mathbf{u}_{\mathrm{T}} \otimes \mathbf{u}_{\mathrm{T}}}{\left\|\mathbf{u}_{\mathrm{T}}\right\|^{2}}\right) \cdot[\mathbf{u}] .
\end{aligned}
$$

Subscripts $\mathrm{N}$ and $\mathrm{T}$ respectively denote the normal and tangential components $\left(\mathbf{R}=R_{\mathrm{N}} \boldsymbol{n}+\mathbf{R}_{\mathrm{T}}\right.$, $\mathbf{R}^{\mathrm{coh}}=R_{\mathrm{N}}^{\mathrm{coh}} \boldsymbol{n}+\mathbf{R}_{\mathrm{T}}^{\mathrm{coh}}$ and $[\mathbf{u}]=u_{\mathrm{N}} \boldsymbol{n}+\mathbf{u}_{\mathrm{T}}$ ), $\boldsymbol{n}$ is the unit normal vector of the cohesive zone (see Fig. 3), $C_{\mathrm{N}}$ and $C_{\mathrm{T}}$ denote respectively the initial normal and tangential stiffness of the perfect interface $(\mathrm{MPa} / \mathrm{m}), I_{K}$ is the indicator function of the set $K, \mu$ is the Coulomb friction coefficient. The surface variable $\beta$, initially introduced by Fremond [16], is governed by Eqs. (4) and (5), where the function $g$ describes the weakening process leading from perfect interface to crack $(\beta=1$ : the interface is undamaged, $0<\beta<1$ : the interface is partially damaged and $\beta=0$ : the interface is fully damaged):

$$
\begin{aligned}
& \beta=\min \left(g(\|[\mathbf{u}]\|), g\left(\|[\mathbf{u}]\|_{\max }\right)\right), \\
& g(x)= \begin{cases}\beta_{0} & \text { if } x \leqslant \delta_{0}, \\
\beta_{0} \frac{\delta_{0}}{x}\left(1-\left(\frac{x-\delta_{0}}{\delta_{c}-\delta_{0}}\right)^{2}\right) & \text { if } \delta_{0}<x<\delta_{c}, \\
0 & \text { if } x \geqslant \delta_{c},\end{cases}
\end{aligned}
$$

where $\delta_{0}=\frac{R_{\max }}{2}\left(\frac{1}{C_{\mathrm{N}}}+\frac{1}{C_{\mathrm{T}}}\right), \delta_{c}=\frac{3}{2}\left(\frac{w}{R_{\max }}+\frac{\delta_{0}}{6}\right), 0 \leqslant \beta_{0} \leqslant 1$ is an initial surface damage, $w$ is a reference fracture energy $\left(\mathrm{J} / \mathrm{m}^{2}\right), R_{\max }$ is the maximum value of the cohesive stress (MPa), $\|[\mathbf{u}]\| \|_{\max }$ is the maximum value reached

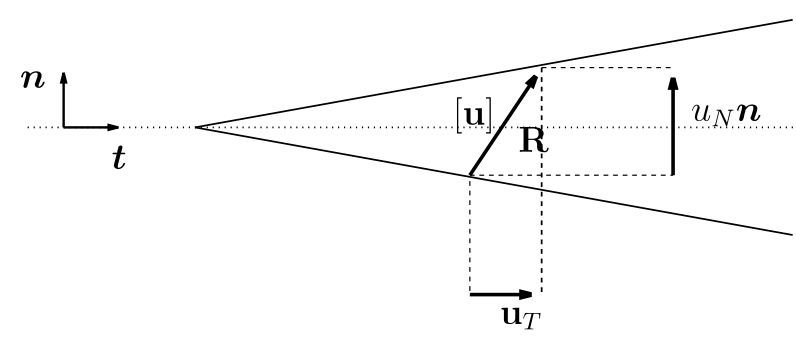

Fig. 3. Notation: stress $\mathbf{R}$ induced by the displacement jump $[\mathbf{u}]$ and normal/tangent decomposition. 
a

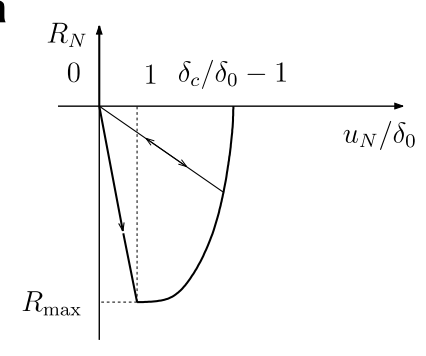

b

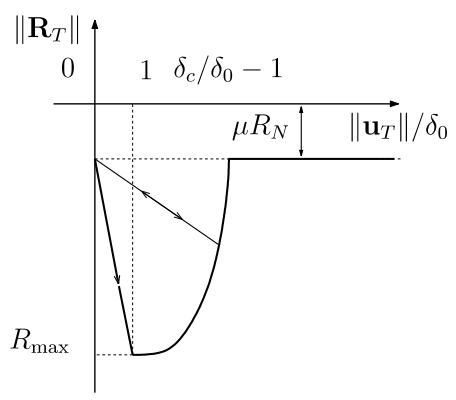

Fig. 4. The 2D FCZM: (a) normal behavior $\left(u_{\mathrm{T}}=0\right)$ and (b) tangent behavior $\left(u_{\mathrm{N}}=0, R_{\mathrm{N}}\right.$ constant).

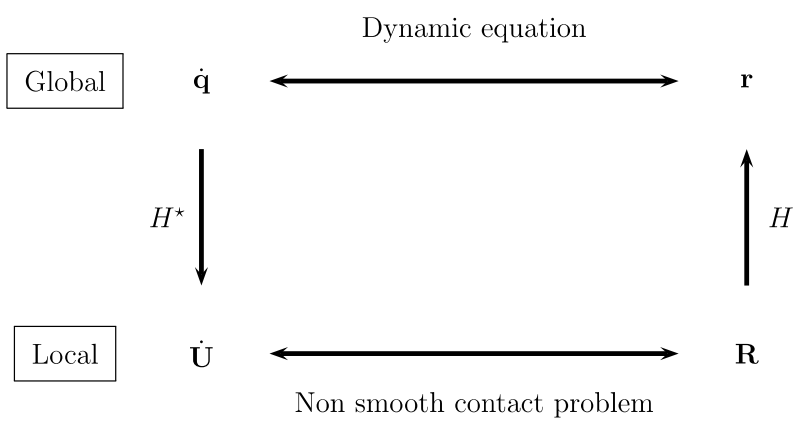

Fig. 5. The standard NSCD algorithm.

by $\|[\mathbf{u}]\|$ during the fracture process. In a $2 \mathrm{D}$ case, Fig. 4 shows respectively (a) the normal behavior (with $\left\|\mathbf{u}_{\mathrm{T}}\right\|=0$ ) and (b) the tangential behavior (with $R_{\mathrm{N}}$ constant) associated with the FCZM (1)-(5).

\subsection{Non-smooth contact dynamics}

Since the FCZMs involve unilateral constraints, the velocity can become discontinuous. These non-regular conditions are treated with the help of the NSCD approach. In this framework, initiated by Moreau [9] and Jean [10], the discrete dynamic equation (6) is treated without any regularization nor penalization, the derivatives are written in a distribution sense using an implicit time integration scheme:

$$
\boldsymbol{M}(\mathbf{q}, t) \cdot \ddot{\mathbf{q}}=\boldsymbol{F}(\mathbf{q}, \dot{\mathbf{q}}, t)+\mathbf{r},
$$

where $\boldsymbol{M ( q}, t)$ is the mass matrix, $\mathbf{q}, \dot{\mathbf{q}}$ and $\ddot{\mathbf{q}}$ are respectively the discrete displacement, velocity and acceleration, $\mathbf{r}$ represents the reaction forces given by the FCZM relationship (1)-(5) and $\boldsymbol{F}(\mathbf{q}, \dot{\mathbf{q}}, t)$ represents the internal and external forces without the contribution of $\mathbf{r}$ [17].

In this framework, a dual level resolution is carried out (for more details, see for example Jean et al. [17]):

- Global level: solving of the discrete dynamic equation (6).

- Local level: solving of the non-smooth contact problem (1)-(5).

At the local level, the local fields at some contact are related to the fields at the global level with the help of a linear mapping $H^{\star}$ (transposed mapping of $H$ ).

The principle of the global to local mapping and the associated variables are summarized in Fig. 5.

\section{Determination of the effective properties: a multibody periodic homogenization framework}

The overall behavior of the composite is numerically determined using a multibody periodic numerical homogenization framework. The numerical homogenization of heterogeneous media consists in computing 
some effective properties over a Representative Volume Element (RVE) [12]. In this work, the RVEs describe the microstructure of nonhomogeneous material with random generation of the heterogeneities distribution.

The homogenization of periodic media uses specific assumptions based on the periodicity of the local fields. The method which provides good approximations of the local behavior of the heterogeneous media is based on linear elasticity on theoretical considerations $[18,11,19,12]$. Some authors have extended this method to an elastic behavior in total Lagrangian finite strain [20,21].

The following deals with an extension of the periodic homogenization framework to the multibody approach. Finite strain within Lagrangian formulation is considered.

\subsection{Extended periodic-cohesive RVE}

A periodic medium is defined by a unit cell and vectors of translation invariance. The unit cell is not uniquely defined and the unit vectors of translation invariance follow the choice of the unit cell [11]. In the multibody case, the RVE includes the cohesive zone associated with the unit volume as indicated in Fig. 6. The fracture between the unit volumes is thus taken into account with the help of mixed relations given by the FCZM. Inside the unit volume, the fracture is described by the multibody approach (Section 2.1).

\subsection{Periodic local fields}

The unit cell problem is obtained considering a periodic medium. In this framework, the deformation gradient field $\boldsymbol{F}=\nabla \mathbf{u}$ and the first Piola-Kirchhoff stress field $\boldsymbol{\Pi}$ are assumed to be periodic with the same period as the medium $[20,21]$. The average fields over the periodic RVE are denoted by $\overline{\boldsymbol{F}}, \bar{\nabla} \mathbf{u}$ and $\overline{\boldsymbol{\Pi}}$, and are performed on the initial configuration $\Omega_{0}$ by:

$$
\bar{f}:=\langle f\rangle_{\Omega_{0}}:=\frac{1}{\left|\Omega_{0}\right|} \int_{\Omega_{0}} f \mathrm{~d} X .
$$

The fields $\boldsymbol{F}, \nabla \mathbf{u}$ and $\boldsymbol{\Pi}$ fluctuate around their average values. The local deformation gradient field can be thus split into an overall field (the field in the periodic RVE if it were homogeneous) and a fluctuation denoted $\nabla \mathbf{u}^{\#}$, which takes the presence of heterogeneities into account. Since fracture is expected, the heterogeneities are not only due to the inclusions but also to the cracks in the structure. The decomposition of the local deformation gradient can be written as:

$$
\nabla \mathbf{u}=\bar{\nabla} \mathbf{u}+\nabla \mathbf{u}^{\#} \quad \text { or } \quad \boldsymbol{F}=\overline{\boldsymbol{F}}+\nabla \mathbf{u}^{\#} .
$$

This relation leads to:

$$
\mathbf{u}^{\#}=\mathbf{x}-\overline{\boldsymbol{F}} \cdot \mathbf{X}
$$

where $\mathbf{x}$ and $\mathbf{X}$ are respectively the current and the initial coordinates.

So, the displacement field $\mathbf{u}^{\#}$ satisfies:

$$
\left\langle\nabla \mathbf{u}^{\#}\right\rangle_{\Omega_{0}}=0 \text {. }
$$

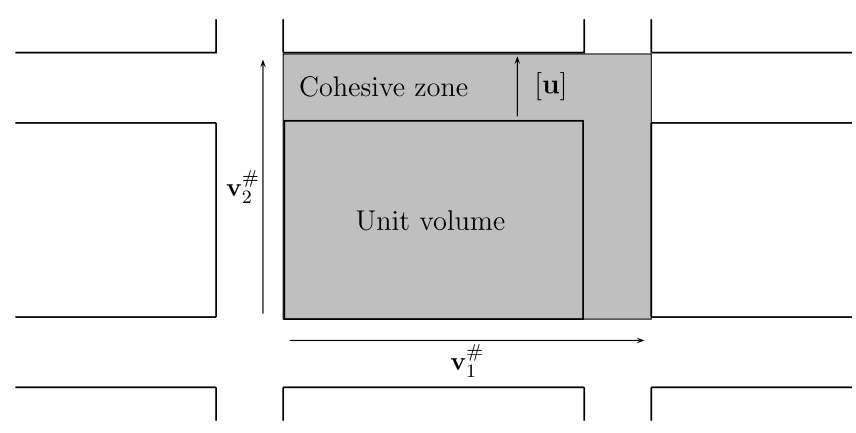

Fig. 6. Composition of the representative volume element: unit volume, vectors of translation invariance and cohesive zone associated with the unit volume. 
This relation and the periodicity of $\nabla \mathbf{u}^{\#}$ imply the periodicity of the displacement field $\mathbf{u}^{\#}$ [22].

The displacement field admits the following decomposition:

$$
\mathbf{u}=(\overline{\boldsymbol{F}}-\mathbf{I}) \cdot \mathbf{X}+\mathbf{u}^{\#} \text {. }
$$

Note that the stress field $\boldsymbol{\Pi}$ is as well assumed periodic and the traction stress $\boldsymbol{\Pi} \cdot \boldsymbol{N}$ is anti-periodic (the normal vectors $\boldsymbol{N}$ at opposite side of the boundary are opposite).

\subsection{Specific boundary conditions}

Let $K_{0}^{e}$ be a mesh, considered as an independent body (see 2.1), of a Finite Element Mesh. The faces of $K_{0}^{e}$ can be splitted into external faces $\partial K_{0}^{e} \cap \partial \Omega_{0}$ (known as boundary faces) and internal faces $\partial K_{0}^{e} \backslash \partial \Omega_{0}$.

The displacement jump is thus decomposed into an internal part and an external part:

$$
[\mathbf{u}]=\sum_{E_{\mathrm{int}}} \mathbf{1}_{E_{\mathrm{int}}}[\mathbf{u}]_{E_{\mathrm{int}}}+\sum_{E_{\mathrm{ext}}} \mathbf{1}_{E_{\mathrm{ext}}}[\mathbf{u}]_{E_{\mathrm{ext}}}
$$

where $E_{\text {int }}$ is an internal face, $E_{\text {ext }}$ is an external face and $\mathbf{1}_{X}$ is the characteristic function of the set $X$ defined as $\mathbf{1}_{X}(k)=1$ if $k \in X$ and $\mathbf{1}_{X}(k)=0$ otherwise.

More precisely,

- for each internal face $E_{\text {int }}$, there exists two bodies $K_{0}^{e}$ and $K_{0}^{f}$ such that $E_{\text {int }}=K_{0}^{e} \cap K_{0}^{f}$ (see Fig. 7). We can define on the internal face $K_{0}^{e}$ :

$$
\begin{aligned}
& \mathbf{u}_{E_{\text {int }}^{-}}^{-}=\text {trace of }\left.\mathbf{u}\right|_{K_{0}^{e}} \text { on } E_{\text {int }}, \\
& \mathbf{u}_{E_{\text {int }}}^{+}=\text {trace of }\left.\mathbf{u}\right|_{K_{0}^{f}} \text { on } E_{\text {int }},
\end{aligned}
$$

and the displacement jump on $K_{0}^{e}$ :

$$
[\mathbf{u}]_{E_{\text {int }}}=\mathbf{u}_{E_{\text {int }}}^{+}-\mathbf{u}_{E_{\text {int }}}^{-},
$$

- periodicity conditions have to be written on the external faces. For each external face $E_{\text {ext }} \subset K_{0}^{e} \cap \partial \Omega_{0}$ of $K_{0}^{e}$, there exists an external face, denoted $E_{\text {ext }}^{\prime}$, which geometrically corresponds to $E_{\text {ext }}$ by periodicity and only one body $K_{0}^{f} \neq K_{0}^{e}$ which contains this face (see Fig. 7). We can define:

$$
\begin{aligned}
& \mathbf{u}_{E_{\mathrm{ext}}}^{-}=\text {trace of }\left.\mathbf{u}\right|_{K_{0}^{e}} \text { on } E_{\mathrm{ext}}, \\
& \mathbf{u}_{E_{\mathrm{ext}}^{\prime}}^{-}=\text {trace of }\left.\mathbf{u}\right|_{K_{0}^{f}} \text { on } E_{\mathrm{ext}}^{\prime} .
\end{aligned}
$$

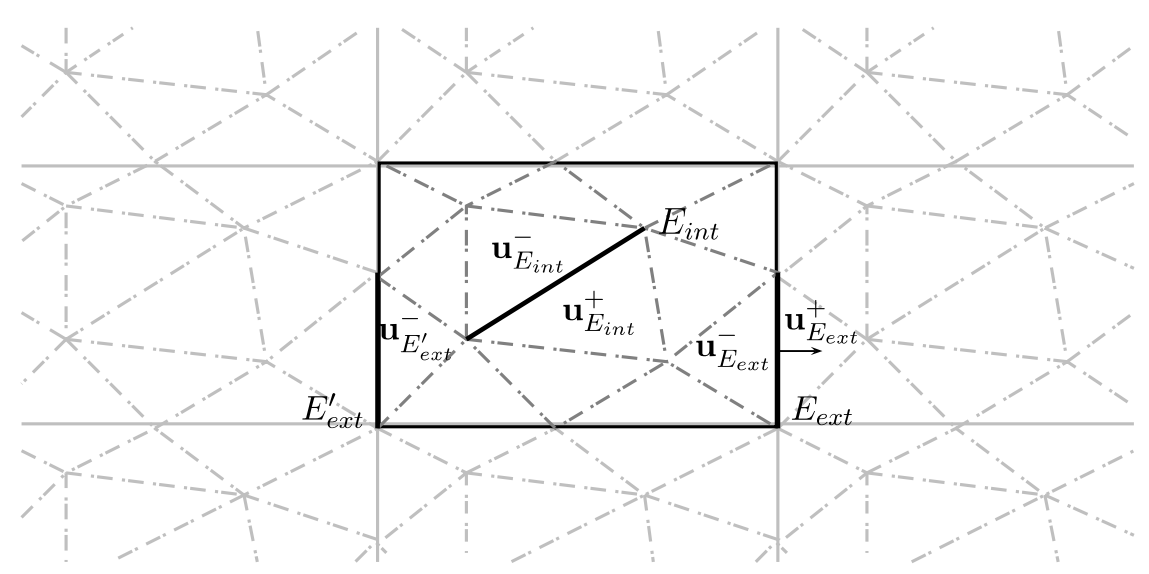

Fig. 7. Finite Element Mesh of a periodic RVE: external faces are periodic. 
Let $\mathbf{u}_{E_{\text {ext }}}^{+}$corresponds to $\mathbf{u}_{E_{\text {ext }}^{\prime}}^{-}$by periodicity. The initial position of $\mathbf{u}_{E_{\text {ext }}^{\prime}}^{-}$and $\mathbf{u}_{E_{\text {ext }}}^{-}$are respectively denoted $\mathbf{X}^{-}$ and $\mathbf{X}^{+}$. We obtain the following relations:

$$
\left\{\begin{array}{l}
\mathbf{u}_{E_{\text {ext }}^{+}}^{+}=(\overline{\boldsymbol{F}}-\mathbf{I}) \cdot \mathbf{X}^{+}+\left(\mathbf{u}_{\boldsymbol{E}_{\text {ext }}}^{+}\right)^{\#} \\
\left(\mathbf{u}_{\boldsymbol{E}_{\text {ext }}^{\prime}}^{-}\right)^{\#}=\mathbf{u}_{\boldsymbol{E}_{\text {ext }}^{\prime}}^{-}-(\overline{\boldsymbol{F}}-\mathbf{I}) \cdot \mathbf{X}^{-} \\
\left(\mathbf{u}_{\boldsymbol{E}_{\text {ext }}}^{+}\right)^{\#}=\left(\mathbf{u}_{\boldsymbol{E}_{\text {ext }}^{\prime}}^{\prime}\right)^{\#} \quad \text { periodicity conditions. }
\end{array}\right.
$$

The displacement jump on the periodic face $E_{\text {ext }}$ is defined by:

$$
[\mathbf{u}]_{E_{\mathrm{ext}}}=\mathbf{u}_{E_{\mathrm{ext}}}^{+}-\mathbf{u}_{E_{\mathrm{ext}}}^{-}=\mathbf{u}_{E_{\mathrm{ext}}^{\prime}}^{-}-\mathbf{u}_{E_{\mathrm{ext}}}^{-}+(\overline{\boldsymbol{F}}-\mathbf{I}) \cdot\left(\mathbf{X}^{+}-\mathbf{X}^{-}\right) .
$$

In a general case, for an internal or an external face denoted $E$, we can write:

$$
[\mathbf{u}]_{E}=\mathbf{u}_{E}^{+}-\mathbf{u}_{E}^{-}=\left(\mathbf{u}_{E}^{+}\right)^{\#}+(\overline{\boldsymbol{F}}-\mathbf{I}) \cdot \mathbf{X}-\left(\left(\mathbf{u}_{\boldsymbol{E}}^{-}\right)^{\#}+(\overline{\boldsymbol{F}}-\mathbf{I}) \cdot \mathbf{X}\right)=\left(\mathbf{u}_{\boldsymbol{E}}^{+}\right)^{\#}-\left(\mathbf{u}_{\boldsymbol{E}}^{-}\right)^{\#}=\left[\mathbf{u}^{\#}\right]_{\boldsymbol{E}} \quad \text { on } \partial \boldsymbol{K}_{\boldsymbol{\theta}}^{\boldsymbol{e}},
$$

where $\mathbf{X}$ is the initial position of $\mathbf{u}_{E}^{+}$and $\mathbf{u}_{E}^{-}$.

Note that the writing of the displacement jump is independent of the choice of the unknowns (standard $\mathbf{u}$ or periodic $\mathbf{u}^{\#}$ ).

The boundary conditions applied on the bodies are mixed relations, given by the FCZM (see 2.2), dependent of the displacement jump (12).

\subsection{Strong formulation}

Neglecting any body force, the local dynamic equilibrium equation in each body $K_{0}^{e}$ is written as:

$$
\nabla \cdot \boldsymbol{\Pi}=\rho \ddot{\mathbf{u}} \quad \text { in } K_{0}^{e},
$$

with boundary conditions related to a cohesive zone model (see Eqs. (1)-(3)):

$$
\boldsymbol{\Pi} \cdot \boldsymbol{N}=\mathbf{R}([\mathbf{u}])=\mathbf{R}\left(\left[\mathbf{u}^{\#}\right]\right) \quad \text { on } \partial K_{0}^{e} .
$$

The unit cell problem is:

find the periodic displacement field $\mathbf{u}^{\#}$, the transformation gradient field $\boldsymbol{F}$ and the stress field $\boldsymbol{\Pi}$ verifying:

- relations for each body $K_{0}^{e}$ :

$$
\begin{cases}\nabla \cdot \boldsymbol{\Pi}=\rho \frac{d^{2}}{d t^{2}}\left(\mathbf{u}^{\#}+(\overline{\boldsymbol{F}}-\mathbf{I}) \cdot \mathbf{X}\right) & \text { in } \boldsymbol{K}_{\boldsymbol{0}}^{\boldsymbol{e},} \\ \boldsymbol{\Pi}^{\prime}(\boldsymbol{F})^{\prime} & (\text { Constitutive law }), \\ \boldsymbol{F}=\overline{\boldsymbol{F}}+\nabla \mathbf{u}^{\#} & \text { in } \boldsymbol{K}_{\boldsymbol{0}}^{\boldsymbol{e},} \\ \boldsymbol{\Pi} \cdot \boldsymbol{N}=\mathbf{R}\left(\left[\mathbf{u}^{\#}\right]\right) & \text { on } \partial \boldsymbol{K}_{\boldsymbol{0}}^{\boldsymbol{e}},\end{cases}
$$

- average relations in $\Omega_{0}$ :

$$
\left\{\begin{array}{l}
\bar{F}_{i j}=\bar{F}_{i j}^{\mathrm{imp}}, \\
\bar{\Pi}_{k l}=\bar{\Pi}_{k l}^{\mathrm{imp}},
\end{array}\right.
$$

where $\bar{F}_{i j}^{\mathrm{imp}}$ are the components of the prescribed macroscopic transformation gradient and $\bar{\Pi}_{k l}^{\mathrm{imp}}$ are the components of the prescribed macroscopic stress, with $i j \neq k l$ and $\{i, j, k, l\} \in\{1,2,3\}$.

We would like to underline, that the set of Eq. (21) is written making the implicit assumption that the standard periodic homogenization method is still valid under dynamic conditions. Without further analysis, dynamics can be here considered as a regularization of the ill-posed static boundary value problem involving softening surface behaviors $[8,23]$. Moreover, note that the problem (21) is subjected to homogeneous conditions (22). Finally, since the total lagrangian formulation is adopted, the averages are written in the initial configuration. 


\subsection{Weak formulation}

In order to obtain a numerical method to compute a solution approximation, a variational statement of our problem is derived, and the broken Sobolev spaces $U$ and $U^{\#}$ are introduced:

$$
\begin{aligned}
& U=\left\{\mathbf{v} \in\left[L^{2}\left(\Omega_{0}\right)\right]^{m},\left.\mathbf{v}\right|_{K_{0}^{e}} \in\left[H^{1}\left(K_{0}^{e}\right)\right]^{m} \forall K_{0}^{e}\right\}, \\
& U^{\#}=\left\{\mathbf{v} \in\left[L^{2}\left(\Omega_{0}\right)\right]^{m},\left.\mathbf{v}\right|_{K_{0}^{e}} \in\left[H^{1}\left(K_{0}^{e}\right)\right]^{m} \forall K_{0}^{e}, \mathbf{v} \text { periodic }\right\},
\end{aligned}
$$

and let $V$ be the space of linear transformation from $\mathbb{R}^{m}$ to $\mathbb{R}^{m}$, where $m$ is the space dimension:

$$
V=\mathscr{L}\left(\mathbb{R}^{m}\right) \text {. }
$$

Consider a kinematically admissible virtual velocity field $\mathbf{v}^{*}$ such that:

$$
\mathbf{v}^{*}=\left(\mathbf{v}^{*}\right)^{\#}+\dot{\boldsymbol{F}}^{*} \cdot \mathbf{X}
$$

with:

$$
\dot{\overline{\boldsymbol{F}}}^{*}=\left\langle\nabla \mathbf{v}^{*}\right\rangle_{\Omega_{0}}
$$

Note that $\mathbf{v}^{*} \in U$ leads to a reduction of the support of basis functions to the current body.

Multiplying the dynamic equation of the system (21) by the virtual velocity field $\mathbf{v}^{*}$, using the decomposition (25), integrating over the whole body $K_{0}^{e}$, adding the equations for each body, applying the Green's theorem to the terms associated with $\mathbf{v}^{*}$ then to the terms associated with $\overline{\boldsymbol{F}}^{*}$, using the definition of the average:

$$
\sum_{e=1}^{\tau} \int_{K_{0}^{e}} \boldsymbol{\Pi}: \dot{\overline{\boldsymbol{F}}}^{*} \mathrm{~d} x=\int_{\Omega_{0}} \boldsymbol{\Pi}: \dot{\overline{\boldsymbol{F}}}^{*} \mathrm{~d} x=\left|\Omega_{0}\right| \overline{\boldsymbol{\Pi}}: \dot{\boldsymbol{F}}^{*},
$$

and taking average loading conditions (22) on each body into account, the following weak unit cell value problem is obtained:

find $\mathbf{u}^{\#} \in U^{\#}$ and $\overline{\boldsymbol{F}} \in \boldsymbol{V}$ such that:

$$
\left\{\begin{array}{l}
\forall\left(\mathbf{v}^{*}\right)^{\#} \in U^{\#}, \\
\sum_{e=1}^{\tau} \int_{\partial K_{0}^{e}} \mathbf{R}\left(\left[\mathbf{u}^{\#}\right]\right) \cdot\left(\mathbf{v}^{*}\right)^{\#} \mathrm{~d} S-\sum_{e=1}^{\tau} \int_{K_{0}^{e}} \boldsymbol{\Pi}: \nabla\left(\mathbf{v}^{*}\right)^{\#} \mathrm{~d} x \\
\quad=\sum_{e=1}^{\tau} \int_{K_{0}^{e}} \rho \frac{d^{2}}{d t^{2}}\left(\mathbf{u}^{\#}+(\overline{\boldsymbol{F}}-\mathbf{I}) \cdot \mathbf{X}\right) \cdot\left(\mathbf{v}^{*}\right)^{\#} \mathrm{~d} \boldsymbol{x} ; \\
\forall \dot{\overline{\boldsymbol{F}}^{*} \in \boldsymbol{V}} \\
\sum_{\boldsymbol{e}=\boldsymbol{I}}^{\tau} \int_{\boldsymbol{K}_{\boldsymbol{e}}^{e}} \boldsymbol{\Pi}: \dot{\overline{\boldsymbol{F}}}^{*} \mathrm{~d} \boldsymbol{x}=\left|\Omega_{\boldsymbol{o}}\right| \overline{\boldsymbol{\Pi}}: \dot{\overline{\boldsymbol{F}}}^{*} .
\end{array}\right.
$$

The variational periodic problem (28) is thus splitted into two equations: (i) a weak formulation written in terms of periodic displacement, (ii) a condition on the macroscopic stress. It should be noticed that the form (28) is not entirely standard due to the use of the Green's theorem for the terms associated with $\dot{\bar{F}}^{*}$, which ensures this convenient decoupling between periodic displacements and macroscopic strain gradient rate.

\subsection{Discrete problem and periodic NSCD}

We replace the infinite dimensional problem (28) by a finite dimensional version: find $\mathbf{u}_{h}^{\#} \in U_{h}^{\#}$ and $\overline{\mathbf{f}}_{h} \in V_{h}$ such that:

$$
\left\{\begin{array}{l}
\forall\left(\mathbf{v}_{h}^{*}\right)^{\#} \in U_{h}^{\#}, \\
\sum_{e=1}^{\tau} \int_{\partial K_{0}^{e}} \mathbf{R}\left(\left[\mathbf{u}_{h}^{\#}\right]\right) \cdot\left(\mathbf{v}_{h}^{*}\right)^{\#} d S-\sum_{e=1}^{\tau} \int_{K_{0}^{e}} \boldsymbol{\Pi}: \nabla\left(\mathbf{v}_{h}^{*}\right)^{\#} \mathrm{~d} x \\
\quad=\sum_{e=1}^{\tau} \int_{K_{0}^{e}} \rho \frac{d^{2}}{d t^{2}}\left(\mathbf{u}_{h}^{\#}+\left(\overline{\mathbf{f}}_{h}-\mathbf{I}\right) \cdot \mathbf{X}\right) \cdot\left(\mathbf{v}_{h}^{*}\right)^{\#} \mathrm{~d} x, \quad \forall \dot{\overline{\boldsymbol{F}}}^{*} \in V_{h}, \\
\sum_{e=1}^{\tau} \int_{K_{0}^{e}} \boldsymbol{\Pi}: \dot{\boldsymbol{F}}^{*} \mathrm{~d} x=\left|\Omega_{0}\right| \overline{\boldsymbol{\Pi}}: \dot{\overline{\boldsymbol{F}}}^{*},
\end{array}\right.
$$


where $U_{h}^{\#}$ and $V_{h}$ are respectively finite dimensional subspaces of $U^{\#}$ and $V$ and built using the standard Finite Element Method:

$$
\begin{aligned}
& V_{h}=\operatorname{vect}\left\{\boldsymbol{\pi}_{j}, j \in\left[1, m^{2}\right], \overline{\boldsymbol{F}} \cdot \boldsymbol{\pi}_{\mathbf{j}} \text { are not prescribed }\right\}, \\
& \boldsymbol{U}_{\boldsymbol{h}}^{\#}=\boldsymbol{v e c t}\left\{\boldsymbol{\varphi}_{\boldsymbol{i}}, \quad \boldsymbol{O} \leqslant \boldsymbol{i} \leqslant \boldsymbol{N}_{\mathbf{u}}^{\text {dof }}\right\},
\end{aligned}
$$

with $\pi_{j}$ the vector basis of the vector space of functions $\mathscr{L}\left(\mathbb{R}^{m}\right), \varphi_{i}$ the basis functions from $\Omega_{0}$ to $\mathbb{R}^{m}$ and $N_{\mathbf{u}}^{\mathrm{dof}}$ the number of free degrees of freedom associated with the periodic displacements.

The integers of the set of indices associated with the vector basis $\pi_{j}$ are re-ordered from 1 to $N \frac{\text { dof }}{F}$, where $N \frac{\text { dof }}{F}$ denote the number of free degrees of freedom associated with the average deformation gradient.

The discrete problem can now be written in the following matrix form:

find $\mathbf{q}^{\#} \in \mathbb{R}^{N_{\mathbf{u}}^{\text {dof }}}$ and $\overline{\mathbf{d}} \in \mathbb{R}^{N \frac{\text { dof }}{\bar{F}}}$ such that:

$$
\begin{aligned}
& \boldsymbol{M}\left(\mathbf{q}^{\#}, t\right) \cdot \ddot{\mathbf{q}}^{\#}+\boldsymbol{N}\left(\mathbf{q}^{\#}, t\right) \cdot \ddot{\overline{\mathbf{d}}}=\boldsymbol{F}\left(\mathbf{q}^{\#}, \dot{\mathbf{q}}^{\#}, \overline{\mathbf{d}}, \dot{\overline{\mathbf{d}}}, t\right)+\mathbf{r}, \\
& 0=\boldsymbol{G}\left(\mathbf{q}^{\#}, \dot{\mathbf{q}}^{\#}, \overline{\mathbf{d}}, \dot{\overline{\mathbf{d}}}, t\right)+\boldsymbol{H}(t),
\end{aligned}
$$

where $\mathbf{q}^{\#}, \dot{\mathbf{q}}^{\#}$ and $\ddot{\mathbf{q}}^{\#}$ are respectively the periodic discrete displacement, velocity and acceleration, $\overline{\mathbf{d}}, \dot{\overline{\mathbf{d}}}$ and $\ddot{\overline{\mathbf{d}}}$ are respectively the discrete average deformation gradient, the first and the second time derivative, $\boldsymbol{M}\left(\mathbf{q}^{\#}, t\right)$ and $N\left(\mathbf{q}^{\#}, t\right)$ are respectively the classical mass matrix and the global concentrated mass matrix, $\mathbf{r}$ represents the reaction forces given by the FCZM relationship, $\boldsymbol{F}(\mathbf{q}, \dot{\mathbf{q}}, t)$ represents the internal and external forces without the contribution of $\mathbf{r}, \boldsymbol{G}\left(\mathbf{q}^{\#}, \dot{\mathbf{q}}^{\#}, \overline{\mathbf{d}}, \dot{\mathbf{d}}, t\right)$ and $\boldsymbol{H}(t)$ represents respectively the macroscopic stress and the macroscopic prescribed stress. The non-standard quantities $\boldsymbol{N}, \mathbf{r}, \boldsymbol{G}$ and $\boldsymbol{H}$ are detailed in Appendix A.

The purpose is now to extend the standard framework of the Non-Smooth Contact Dynamics approach (see 2.3) to the periodic case. Following [9,17], the discrete dynamic system (31) is rewritten in a distribution sense and integrating over the time interval $\left.] t_{i}, t_{i+1}\right]$ using an implicit time integration scheme (see Appendix B). Denoting by a subscript $i$ quantities at a time $t_{i}$ and by $i+1$ quantities at time $t_{i+1}$, assuming that the mass matrices $\boldsymbol{M}\left(\mathbf{q}^{\#}, t\right)$ and $\boldsymbol{N}\left(\mathbf{q}^{\#}, t\right)$ vary slowly with $\mathbf{q}^{\#}$, the use of a $\theta$-method (with $\theta=1 / 2$ in the following) leads to:

$$
\begin{aligned}
& \boldsymbol{M}\left(\mathbf{q}_{i+1}^{\#}, t_{i+1}\right) \cdot\left(\dot{\mathbf{q}}_{i+1}^{\#}-\dot{\mathbf{q}}_{i}^{\#}\right)+\boldsymbol{N}\left(\mathbf{q}_{i+1}^{\#}, t_{i+1}\right) \cdot\left(\dot{\overline{\mathbf{d}}}_{i+1}-\dot{\overline{\mathbf{d}}}_{i}\right)=h_{i}\left\{(1-\theta) \boldsymbol{F}_{i}+\theta \boldsymbol{F}_{i+1}\right\}+h_{i} \mathbf{r}_{i+1}, \\
& 0=h_{i}\left\{(1-\theta) \boldsymbol{G}_{i}+\theta \boldsymbol{G}_{i+1}\right\}+h_{i}\left\{(1-\theta) \boldsymbol{H}_{i}+\theta \boldsymbol{H}_{i+1}\right\},
\end{aligned}
$$

and

$$
\begin{aligned}
& \mathbf{q}_{i+1}^{\#}=\mathbf{q}_{i}^{\#}+h_{i}\left\{(1-\theta) \dot{\mathbf{q}}_{i}^{\#}+\theta \dot{\mathbf{q}}_{i+1}^{\#}\right\}, \\
& \overline{\mathbf{d}}_{i+1}=\overline{\mathbf{d}}_{i}+h_{i}\left\{(1-\theta) \dot{\overline{\mathbf{d}}}_{i}+\theta \dot{\overline{\mathbf{d}}}_{i+1}\right\},
\end{aligned}
$$

where the mean value impulse denoted $h_{i} \mathbf{r}_{i+1}$ emerges as primary unknown:

$$
\int_{]_{i}, t_{i+1}\right]} \mathbf{r d} v=h_{i} \mathbf{r}_{i+1} .
$$

The primary unknowns of the discrete dynamic problem are the approximation of the periodic velocity $\dot{\mathbf{q}}_{i+1}^{\#}$, the first order time derivative of the average deformation gradient $\dot{\mathbf{d}}_{i+1}$ and the impulse $h_{i} \mathbf{r}_{i+1}$. The periodic displacement $\mathbf{q}_{i+1}^{\#}$ and the average of the deformation gradient $\overline{\mathbf{d}}_{i+1}$ are considered as secondary variables.

Solving the nonlinear system (32) with the help of a Newton-Raphson algorithm (iterations are denoted by superscript $k$, see Appendix $\mathrm{C}$ ), one obtains:

$$
\mathbf{A}^{k} \cdot\left\{\begin{array}{c}
\left(\dot{\mathbf{q}}_{i+1}^{\#}\right)^{k+1}-\left(\dot{\mathbf{q}}_{\text {free }}^{\#}\right)^{k+1} \\
\dot{\overline{\mathbf{d}}}_{i+1}^{k+1}-\dot{\overline{\mathbf{d}}}_{\text {free }}^{k+1}
\end{array}\right\}=\left\{\begin{array}{c}
h_{i} \mathbf{r}_{i+1}^{k+1} \\
0
\end{array}\right\},
$$

where the so-called 'free' unknowns $\dot{\mathbf{q}}_{\text {free }}^{\#}$ and $\dot{\overline{\mathbf{d}}}_{\text {free }}$ are respectively the values of the discrete periodic velocity and the discrete first time derivative of the average of the deformation gradient when $\mathbf{r}_{i+1}^{k+1}=0$, and are solution of the following system: 


$$
\mathbf{A}^{k} \cdot\left\{\begin{array}{c}
\left(\dot{\mathbf{q}}_{\text {free }}^{\#}\right)^{k+1}-\left(\dot{\mathbf{q}}_{i+1}^{\#}\right)^{k} \\
\dot{\overline{\mathbf{d}}}_{\text {free }}^{k+1}-\dot{\overline{\mathbf{d}}}_{i+1}^{k}
\end{array}\right\}=\left\{\begin{array}{c}
{\left[-\boldsymbol{M}\left(\mathbf{q}_{i+1}^{\#}, t_{i+1}\right) \cdot\left(\left(\dot{\mathbf{q}}_{i+1}^{\#}\right)^{k}\right)-\dot{\mathbf{q}}_{i}^{\#}\right)} \\
\left.-\boldsymbol{N}\left(\mathbf{q}_{i+1}^{\#}, t_{i+1}\right) \cdot\left(\dot{\overline{\mathbf{d}}}_{i+1}^{k}-\dot{\overline{\mathbf{d}}}_{i}\right)+\mathscr{H}_{\theta}(\boldsymbol{F})\right] \\
\mathscr{H}_{\theta}(\boldsymbol{G})+\mathscr{H}_{\theta}(\boldsymbol{H})
\end{array}\right\}
$$

with

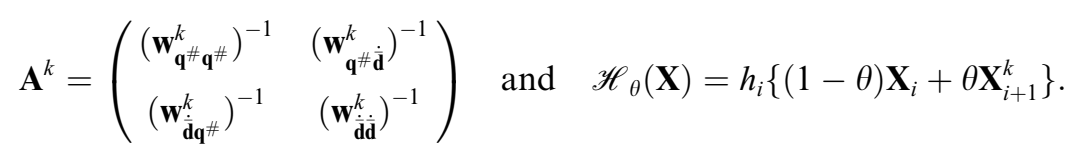

At this stage, the unknowns of the problem are $\dot{\mathbf{q}}^{\#}$ and $\dot{\overline{\mathbf{d}}}$. A change of variable permits to recover the discrete velocity $\dot{\mathbf{q}}$ as primary unknown from $\dot{\mathbf{q}}^{\#}$ and $\dot{\mathbf{d}}$ :

$$
\dot{\mathbf{q}}=P^{-1}\left\{\begin{array}{c}
\dot{\mathbf{q}}^{\#} \\
\dot{\overline{\mathbf{d}}}
\end{array}\right\}
$$

where $P$ is a mapping such that:

$$
\left\{\begin{array}{l}
P: \mathbb{R}^{N_{\mathrm{u}}^{\mathrm{dof}}} \rightarrow \mathbb{R}^{N_{\mathrm{u}}^{\text {dof }}+N_{\overline{\boldsymbol{F}}}^{\mathrm{dof}}} \\
\dot{\mathbf{q}} \mapsto\left\{\begin{array}{c}
\dot{\mathbf{q}}^{\#} \\
\dot{\overline{\mathbf{d}}}
\end{array}\right\}=\left\{\begin{array}{c}
\dot{\mathbf{q}}-\mathbf{R e l}\langle\nabla \dot{\mathbf{q}}\rangle_{\mathrm{d}} \cdot \mathbf{X}_{\mathrm{d}} \\
\langle\nabla \dot{\mathbf{q}}\rangle_{\mathrm{d}}
\end{array}\right\},
\end{array}\right.
$$

with $\mathbf{R e l} \in \mathbb{R}^{N_{\mathbf{u}}^{\text {dof }}} \times \mathbb{R}^{m}$ is a discrete mapping such that the periodic velocity is given by $\dot{\mathbf{q}}^{\#}=\dot{\mathbf{q}}-\operatorname{Rel}\langle\nabla \dot{\mathbf{q}}\rangle_{\mathrm{d}} \cdot \mathbf{X}_{\mathrm{d}}$, $\mathbf{X}_{\mathrm{d}}$ is the discrete position vector and $\langle\cdot\rangle_{\mathrm{d}}: \mathbb{R}^{N_{\mathrm{u}}^{\text {dof }}} \times \mathbb{R}^{m} \rightarrow \mathbb{R}^{N_{\bar{F}}^{\text {dof }}}$ is the discrete average mapping over $\Omega_{0}$.

At the iteration $k+1$, the discrete velocity and the discrete free velocity write:

$$
\dot{\mathbf{q}}_{i+1}^{k+1}=P^{-1}\left\{\begin{array}{c}
\dot{\mathbf{q}}_{i+1}^{k+1} \\
\dot{\mathbf{d}}_{i+1}^{k+1}
\end{array}\right\}, \quad \dot{\mathbf{q}}_{\text {free }}^{k+1}=P^{-1}\left\{\begin{array}{c}
\left(\dot{\mathbf{q}}_{\text {free }}^{\#}\right)^{k+1} \\
\dot{\overline{\mathbf{d}}}_{\text {free }}^{k+1}
\end{array}\right\} .
$$

Note that the action-reaction principle leads to: $\langle\nabla \mathbf{r}\rangle_{\mathrm{d}}=0$.

The change of variable (38) is applied to the iteration matrix (36):

$$
\left(\mathbf{w}^{k}\right)^{-1}:=P^{-1} \mathbf{A}^{k} P \text {. }
$$

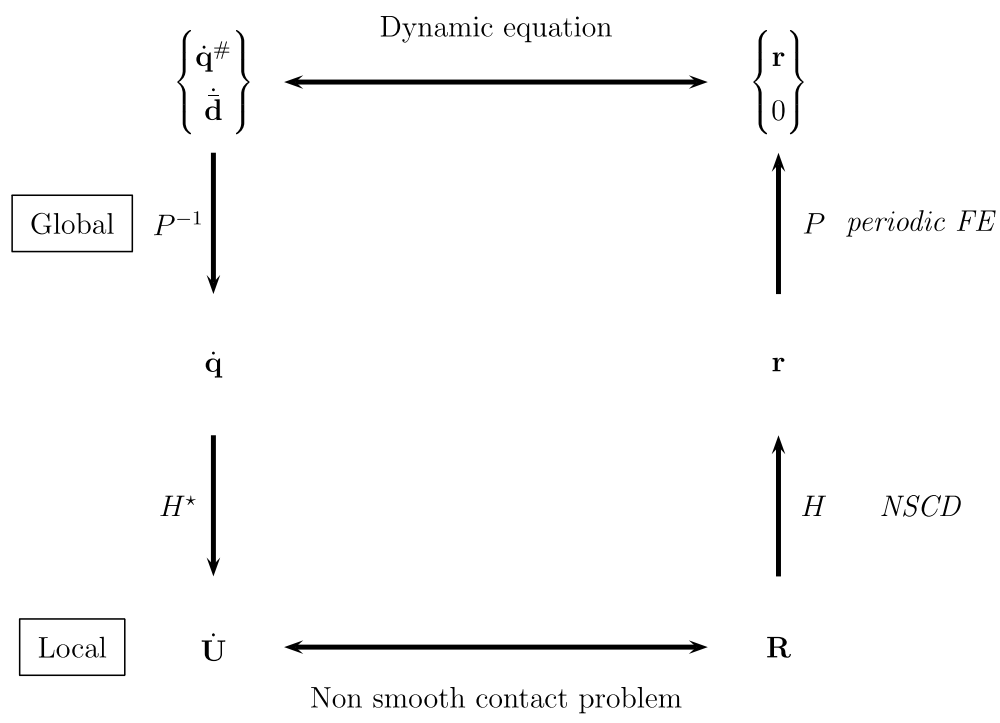

Fig. 8. The NSCD algorithm extended to the periodic formulation. 
The local problem at the contact level is thus solved using the standard NSCD algorithm without any modification (see $[17,8]$ for details about the resolution of the local problem, including FCZM). The two field periodic formulation can thus be shown as a simple extension to the global level of the NSCD algorithm, that is to say the resolution of the periodic Finite Element problem.

The two level resolution of the periodic problem is summarized in Fig. 8:

- at global level: the discrete dynamic equation written in terms of discrete periodic velocity $\dot{\mathbf{q}}^{\#}$ and of first derivative average deformation gradient $\dot{\overline{\mathbf{d}}}$ (Eqs. (35), (36)) is solved,

- at local level: the standard local NSCD resolution is used.

\subsection{Software}

The software architecture respects the local/global levels of the above NSCD two field extension approach by the coupling of three libraries with Object-Oriented Programming [24]:

- LMGC90 is a library dedicated to the contact problems [13],

- PELICANS is a library dedicated to periodic Finite Elements [14],

- MatLib is a material constitutive models library [15].

The software, called 'X-per', allows to simulate, at finite strain, the fracture of heterogeneous materials and periodic structure from the crack initiation to non-smooth post-fracture behavior. In the next section, the ability of this software and of the numerical framework is illustrated on the fracture of metal matrix composites from the nuclear industry (elastoplastic matrix with brittle inclusions).

\section{Applications}

In what follows, the finite element discretization is based on linear displacement triangular elements that are arranged in a "crossed-triangle" quadrilateral pattern. The analysis considers 2D plane-strain conditions. The considered metal matrix composite is representative of hydrided Zircaloy-based alloys which compose cladding of nuclear fuel rods in Pressurized Water Reactor (PWR), at high burnup.

The considered microstructure is thus composed of a metal matrix (Zircaloy-4) and rectangular aligned inclusions (zirconium hydrides). The Zircaloy-4 behavior is assumed to be elastoplastic ( $\mathrm{J} 2$ plasticity, Young Modulus $E=99 \mathrm{GPa}$, Poisson's ratio $v=0.325$, Yield in tension $\sigma_{0}=450 \mathrm{MPa}$, Hardening Modulus $\left.H_{\mathrm{Y}}=850 \mathrm{MPa}\right)[25,26]$ and hydrides to be neo-Hookean $(E=135 \mathrm{GPa}, v=0.32)[27,28]$. The density of the Zircaloy- 4 and the hydrides are identical and equal to $7800 \mathrm{~kg} / \mathrm{m}^{3}$. The FCZM coefficients of Zircaloy4, zirconium hydrides and Zircaloy-hydride interface are respectively: $C_{\mathrm{N}}^{\mathrm{Zr}}=2 \times 10^{18} \mathrm{~Pa} / \mathrm{m}, w^{\mathrm{Zr}}=1 \mathrm{~J} / \mathrm{m}^{2}$ and $R_{\max }^{\mathrm{Zr}}=2.4 \sigma_{0}, \quad C_{\mathrm{N}}^{\mathrm{ZrH}}=2 C_{\mathrm{N}}^{\mathrm{Zr}}, \quad w^{\mathrm{ZrH}}=0.8 w^{\mathrm{Zr}}$ and $R_{\max }^{\mathrm{ZrH}}=3 \sigma_{0}, \quad C_{\mathrm{N}}^{\mathrm{Zr}-\mathrm{ZrH}}=2 C_{\mathrm{N}}^{\mathrm{Zr}}, \quad w^{\mathrm{Zr}-\mathrm{ZrH}}=0.8 w^{\mathrm{Zr}}$ and $R_{\max }^{\mathrm{Zr}-\mathrm{ZrH}}=3 \sigma_{0}$. The bonding strength value between the two phases is thus considered as 'strong'. Moreover, we assume a low friction coefficient $\mu=0.05$ and same compliance for the normal and tangential behaviors $C_{\mathrm{N}}=C_{\mathrm{T}}$.

\subsection{Unit cell}

Two square RVEs (length $L=20 \mu \mathrm{m}$ ), composed by a rectangular inclusion (width $=2 \mu \mathrm{m}$, length $=10 \mu \mathrm{m}$ ) included in a metal matrix, are chosen (see Fig. 9, up). In the first choice, the rectangular inclusion is centered in the RVE. The second RVE contains an off-centered inclusion which is decomposed using the periodicity conditions. A macroscopic strain gradient rate is prescribed along the direction of the aligned inclusions $\left(\overline{\boldsymbol{F}}_{x x}=0.18 c_{\mathrm{d}} / L\right.$ where $c_{\mathrm{d}}$ is the dilatational wave speed of the Zircaloy).

\subsubsection{Illustration of the result independence with respect to the unit cell}

In this section, the independence of the overall behavior of a periodic heterogeneous medium with respect to the unit cell - well known in the case of standard periodic homogenization - is checked in the case of the 

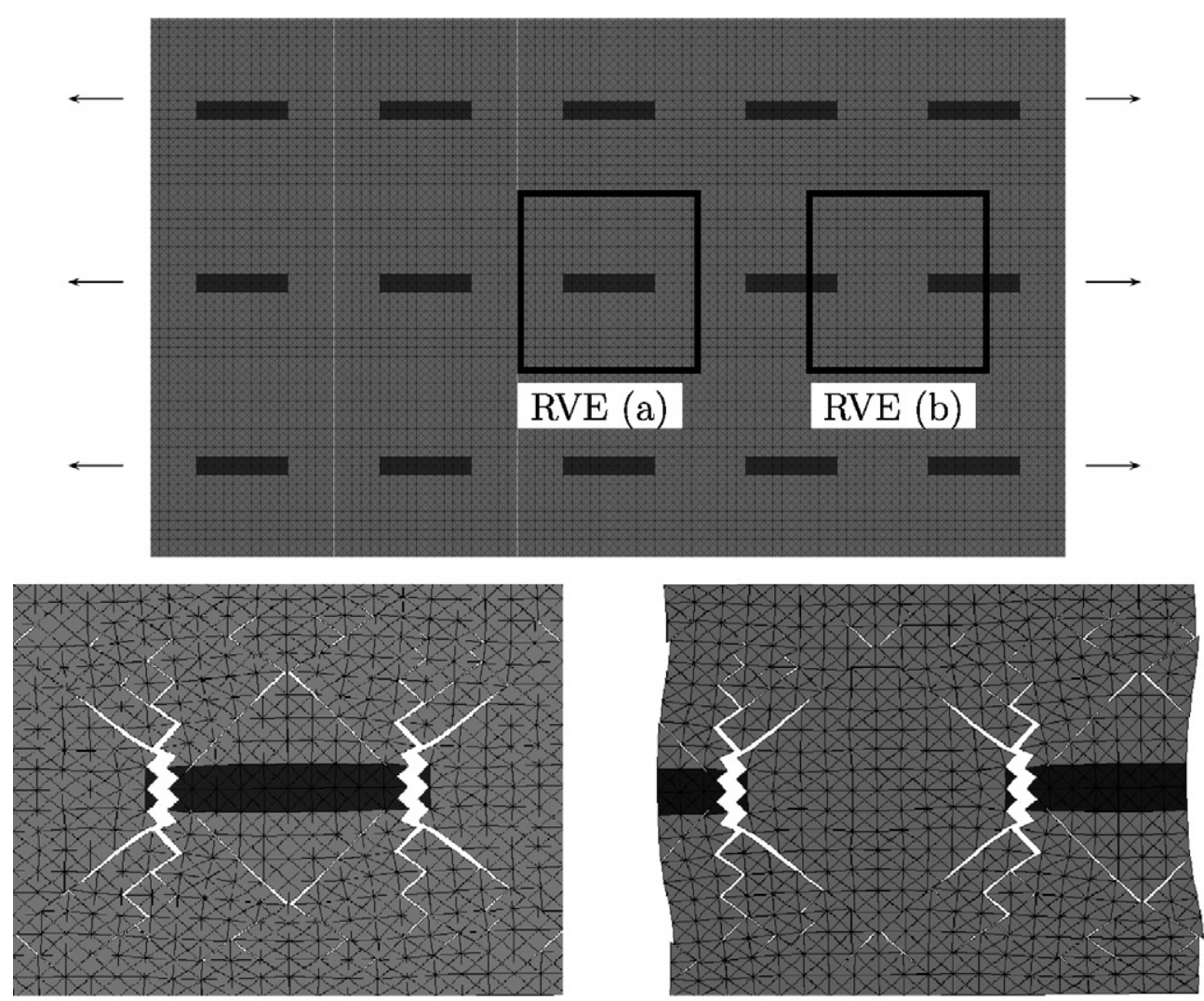

RVE (a): centered inclusion

RVE (b): off-centered inclusion

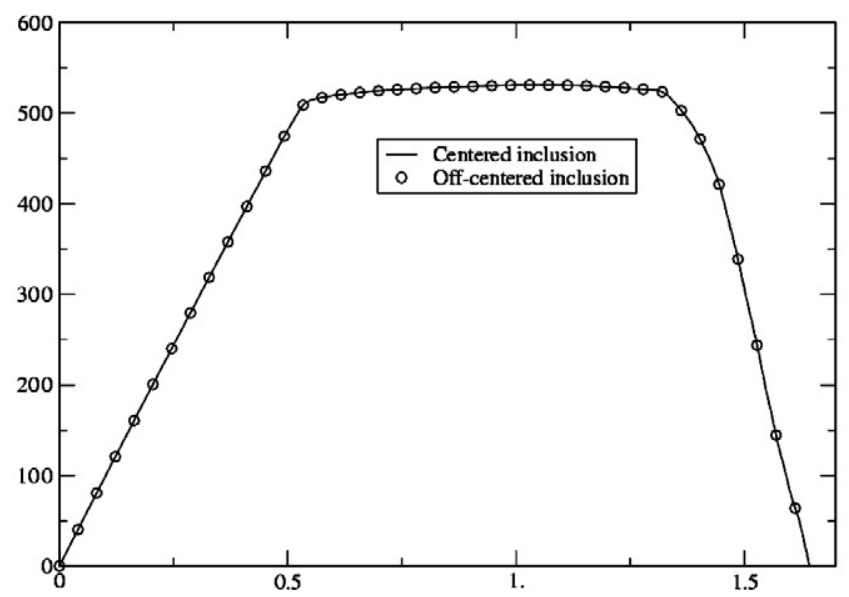

Fig. 9. Illustration of the unit cell independence. Up: periodic medium and two choices of RVE; mid: rupture features $(1 \mu \mathrm{m}$ mesh size in a 'crossed-triangle' quadrilateral pattern); bottom: overall stress ( $\mathrm{MPa})$-strain (\%) curves.

present two field multibody extension. Fig. 9 (mid) shows the rupture features of the considered RVEs. The cracks initiate in the brittle inclusion and propagate through the elastoplastic matrix. We can note that the shape of the crack network is similar in the two cases, and, within the numerical accuracy, the overall stress/strain curves are identical (Fig. 9, bottom). The two choices of the RVE are thus equivalent, as predicted by the standard theory [11]. 
The test example validates the fact that the periodicity conditions are correctly taken into account in the numerical framework and its implementation in the developed software.

\subsubsection{Overall response and energies invoked during the fracture process}

Fig. 10 shows the overall stress-strain curve and the energies (elastic, plastic, 'surface', kinetic and total) invoked during the fracture process. The surface energy is composed by the stored energy and the dissipated energy along the cohesive zones during the process.

During the overall elastic process, the elastic strain energy increases quadratically with respect to the macroscopic strain and the other energies are almost negligible. During the overall plastic process, the elastic strain energy stays quasi-stationary and the plastic energy has a quasi-linear variation (quasi-perfect plastic overall behavior). During the progressive onset of the fracture, the elastic strain energy is released, while the surface energy increases up to $25 \%$ of the total energy and the kinetic energy has very slight variations.

This kinetic energy remains almost negligible (and constant) during the entire fracture process and does not disturb the overall energy balance. The stress-strain curve is smooth. These last two points seems to confirm the hypothesis that the periodic homogenization method stays valid in the considered dynamic conditions (see $\S 3.4$ ). In particular, it seems that prescribing homogeneous strain over the whole domain combined with periodicity conditions allows to limit some dynamic effects such as high frequency modes or wave reflections on the boundary of the domain (see Fig. 11).

\subsubsection{Sensitivity to the size element}

As previously announced, this paper does not have the goal to fully address the problem of the sensitivity of the cohesive-volumetric finite element scheme on the size element. However, since the present numerical method is devoted to the overall fracture properties, this section illustrates that, whatever the main crack path (slightly) depends on the element size, the macroscopic fracture energy stays constant with respect to the size element when (i) the 'standard' finite element method has reached its convergence, (ii) the compliance of the cohesive zone model is suitably adapted to the size element.

The first illustration is given by a mesh refinement (from $1 \mu \mathrm{m}$ size element to $0.5 \mu \mathrm{m}$ ) of the 'centeredoff-centered' case treated in the previous section. The comparison between the corresponding rupture features (Figs. 9 and 12) sketches that the crack path dependence on the mesh refinement is lower in the present case (periodic boundary conditions) than in the case of structure calculus (see e.g. [30]).

The second illustration concerns the independence of the overall fracture properties on the size element. For the sake of simplicity, this illustration was carried out on the previous 'centered inclusion' example, involving a brittle elastic matrix. For this section only, the CFZMs related to the bulk materials (resp. to the interface inclusion/matrix) were thus assumed to be weak (resp. strong): $w^{\mathrm{Zr}}=0.05 \mathrm{~J} / \mathrm{m}^{2}$, and $R_{\max }^{\mathrm{Zr}}=241 \mathrm{MPa}$; $w^{\mathrm{ZrH}}=0.04 \mathrm{~J} / \mathrm{m}^{2}$, and $R_{\max }^{\mathrm{ZrH}}=304 \mathrm{MPa} ; w^{\mathrm{Zr}-\mathrm{ZrH}}=0.5 \mathrm{~J} / \mathrm{m}^{2}$ and $R_{\max }^{\mathrm{Zr}-\mathrm{ZrH}}=1076 \mathrm{MPa}$. Moreover, the initial

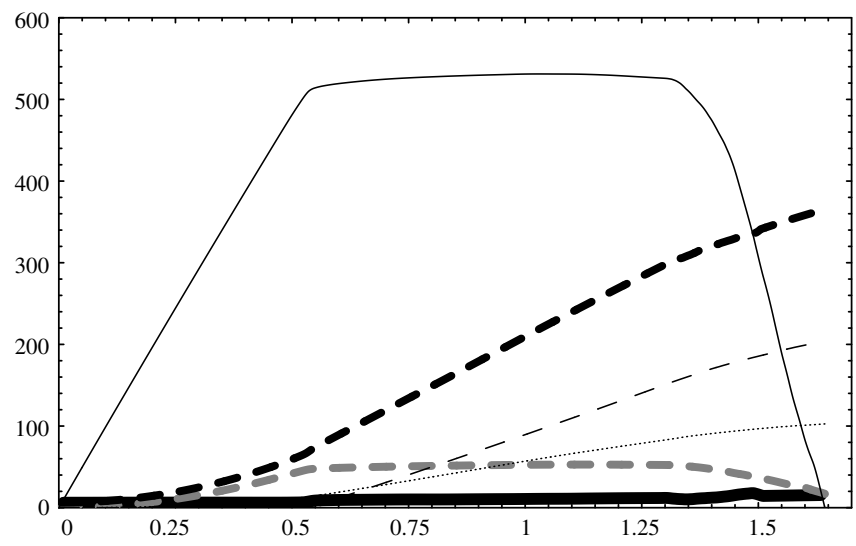

Fig. 10. Overall stress (MPa)- strain (\%) curve (thin line); energies during the fracture process (J): total energy (thick dashed dark line), elastic energy (thick dashed light line), plastic energy (thin dashed line), surface energy (dotted line) and kinetic energy (thick line). 


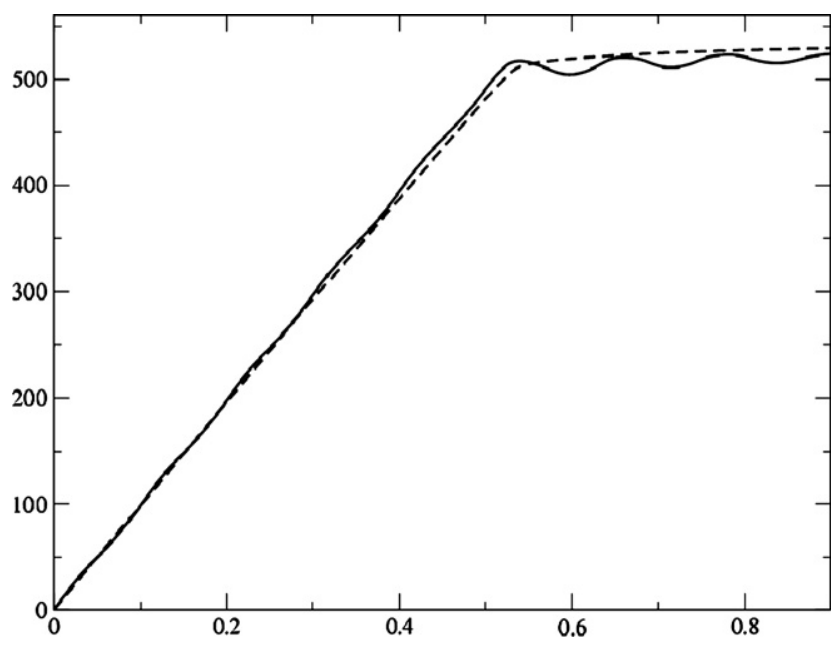

Fig. 11. Effect of periodic boundary conditions: comparison between a periodic simulation (i.e. present method, same simulation as Fig. 10) (dashed line) and the same simulation with standard boundary conditions (i.e. unidirectional traction with standard Dirichlet $\left(\dot{v}_{x}\right)$ and homogeneous Neumann $\left(\sigma_{y y}=0\right)$ boundary conditions) (thick line).
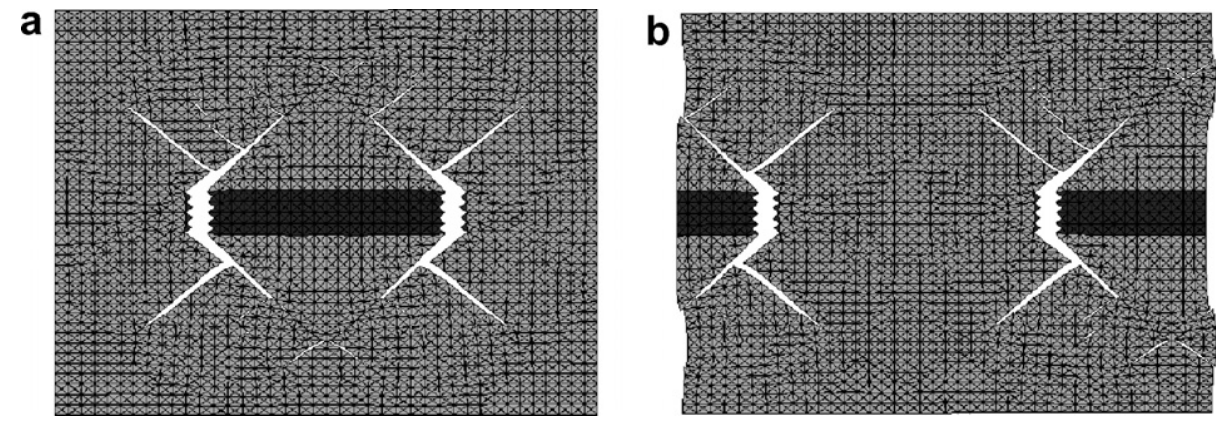

Fig. 12. Rupture features of the two RVEs with $0.5 \mu \mathrm{m}$ mesh size: (a) centered inclusion (b) off-centered inclusion.

stiffness of the CZMs $\left(C_{\mathrm{N}}=C_{\mathrm{T}}\right)$ are taken to be dependent of the mesh size $\left(L_{\text {mesh }}\right)$ with respect to a micromechanical-based criterion (the smaller the size element, the larger the stiffness of CZMs), initially proposed by [29]. This criterion, briefly recalled here, consists in determining the Hashin and Strickman lower bound related to an elastic medium made of penny shaped elastic inclusions (the cohesive zone before any surface damage) randomly distributed in space and in orientation in an isotropic matrix (the bulk material). Denoting by $Z$ the density of the penny shaped inclusions, and combining the overall bulk and shear moduli associated to this lower bound, the overall Young modulus of the medium $\bar{E}$ reads:

$$
\frac{\bar{E}}{E}=\frac{\xi}{1+\xi}, \quad \text { where } \xi=\frac{5}{1+\frac{4}{3} \frac{C_{\mathrm{N}}}{C_{\mathrm{T}}}} \frac{C_{\mathrm{N}}}{E Z},
$$

and where $E$ denotes the Young modulus of the bulk material. Specifying this equation to the case $C_{\mathrm{N}}=C_{\mathrm{T}}$, and to crossed-triangle quadrilateral pattern meshes (in that case $Z=2(1+\sqrt{2}) / L_{\text {mesh }}$ and the bound becomes an estimate), one obtains an applied criterion, which ensures that, when

$$
C_{\mathrm{N}} L_{\mathrm{mesh}} / E \geqslant 20
$$

the added compliance due to the presence of cohesive zones disturbs for less than $10 \%$ the overall elasticity resulting from the finite element discretization. In the present example, this criterion is strictly ensured for the matrix $\left(C_{\mathrm{N}}^{\mathrm{Zr}} L_{\text {mesh }} / E^{\mathrm{Zr}}=21\right)$ and overestimated for the inclusion $\left(C_{\mathrm{N}}^{\mathrm{ZrH}} L_{\mathrm{mesh}} / E^{\mathrm{ZrH}}=29, C_{\mathrm{N}}^{\mathrm{Zr}-\mathrm{ZrH}}=C_{\mathrm{N}}^{\mathrm{ZrH}}\right)$. 


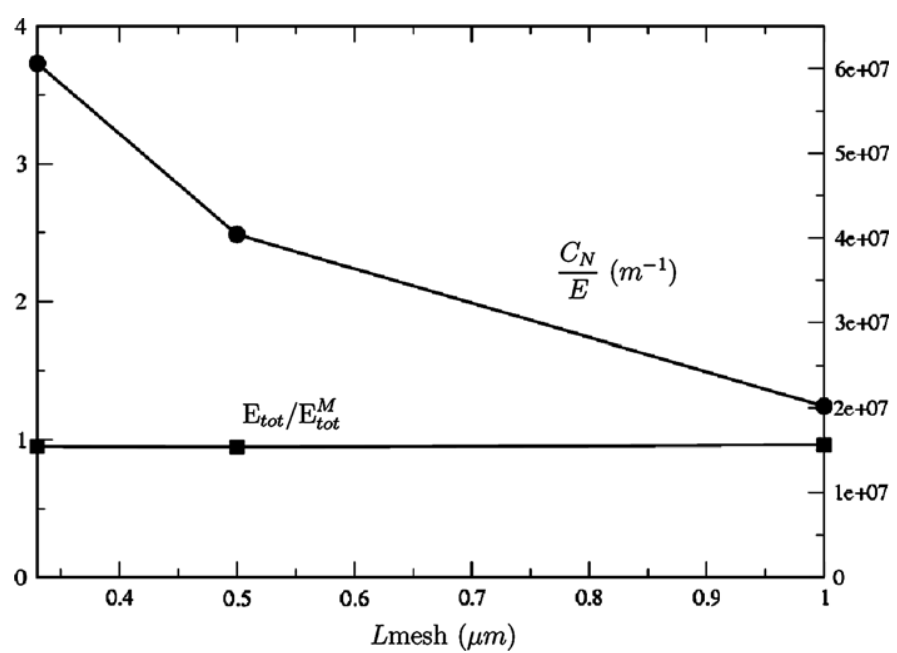

Fig. 13. Macroscopic energy/macroscopic energy of matrix and initial CZM stiffness of the matrix versus the mesh size ( $\mu$ m) for three fraction of inclusions.

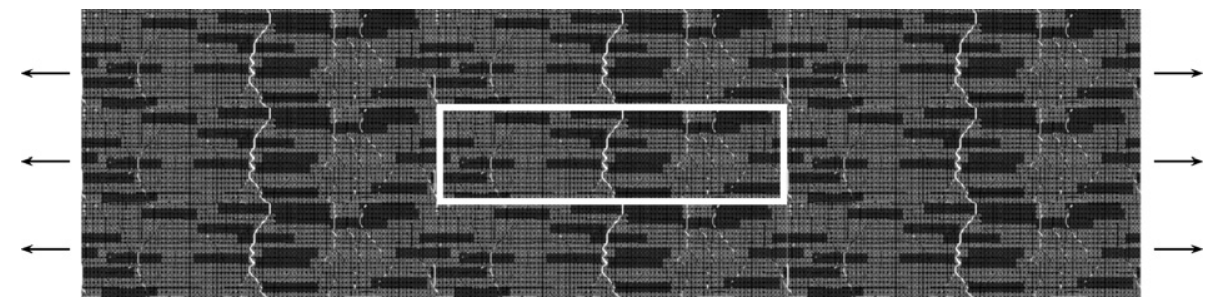

Fig. 14. Broken RVE ( $30 \%$ of inclusions) reproduced by periodicity (nine RVEs).

\section{a}

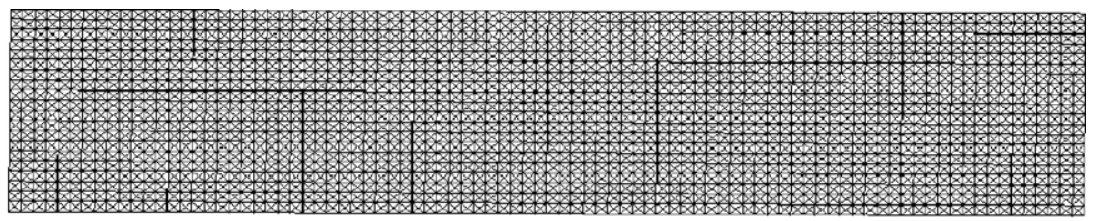

$+$

b
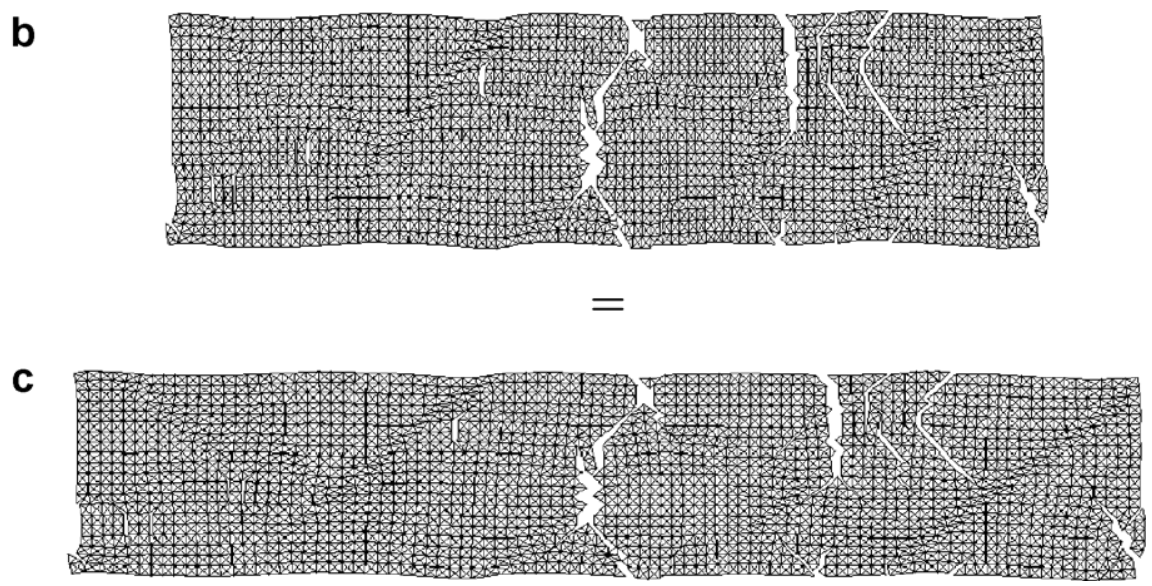

Fig. 15. Decoupling of the global strain (c) of the periodic RVE on a homogeneous part (a) and a corrective part (b). This correction takes the presence of heterogeneities (inclusions and fracture) into account. 
Fig. 13 thus shows that, in the treated case, the overall fracture energy is independent of the mesh size as soon as this criterion is respected and the mesh size is sufficiently small to consider that the Finite Element simulations are converged.

\subsection{Fracture of hydrided Zircaloy-4}

This section deals with an application from the nuclear industry: the fracture of the heterogenous material, which composes the highly irradiated nuclear fuel rod cladding. Zircaloy cladding tubes at high burnup are considered as a bimaterial which is formed by hydride inclusions (brittle) surrounded by a metallic matrix. The inclusions are assumed to be rectangular (width $=2 \mu \mathrm{m}$, length $=10 \mu \mathrm{m}$ ), aligned and randomly distributed in space (see, for example, Fig. 14 for 30\% of hydrogen content). The RVEs are rectangular with $20 \mu \mathrm{m}$ width and $L=88 \mu \mathrm{m}$ length. A macroscopic strain gradient rate is prescribed along the direction of the aligned inclusions $\left(\dot{\boldsymbol{F}}_{x x}=0.02 c_{\mathrm{d}} / L\right)$.

\subsubsection{Weakening effects of brittle inclusions}

Fig. 14 shows the fractured RVE with $30 \%$ of hydrides reproduced by periodicity. The cracks initiate in the area with high contents of hydrogen and propagate into the Zircaloy matrix.

The deformation gradient field split (see 3.2) is illustrated in Fig. 15. In particular, Fig. 15a shows the deformed periodic RVE if it were homogeneous, i.e. without any heterogeneities. The correction (Fig. 15b)
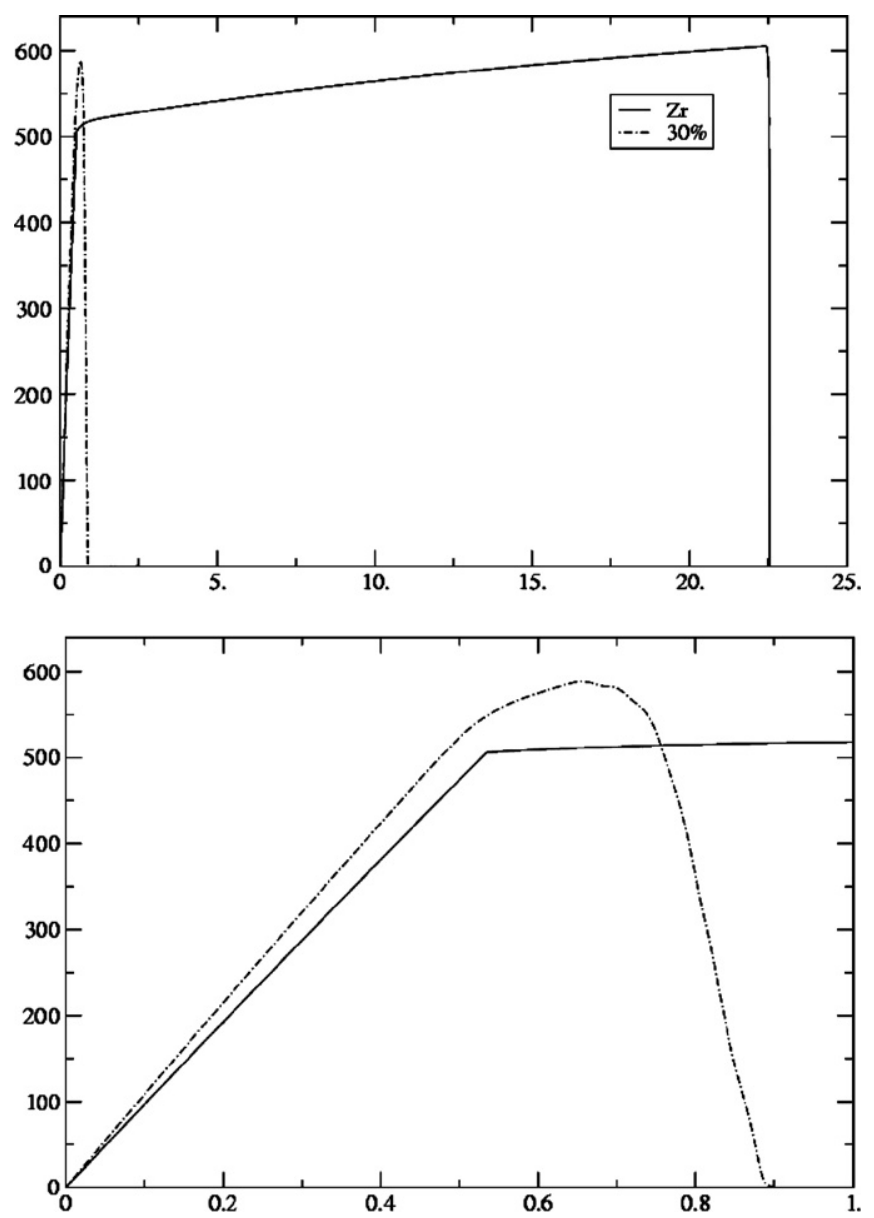

Fig. 16. Overall stress (MPa)-strain (\%) curve of the Zircaloy-4 (solid curve) and the $30 \%$ hydrided Zircaloy-4 (dashed curve) (top) with a zoom at small strain (bottom). 
takes heterogeneities into account. The heterogeneities are given by the heterogeneous microstructure (inclusions) and by the cracks in the structure. The global behavior (Fig. 15c) is thus obtained by summing these two strains (see Eq. (8)).

The stress-strain curves for the Zircaloy- 4 without hydrides and the $30 \%$ hydrided Zircaloy-4 are plotted in Fig. 16. First, the volumetric behavior (elastoplastic) associated with the FCZM (softening function) lead to a damage elastoplasticity behavior for the Zircaloy-4 without any hydride. The introduction of $30 \%$ volume fraction of inclusions reduces the overall fracture energy by $98 \%$. In this case, the overall behavior of the hydrided Zircaloy- 4 is brittle. These results show the deleterious influence of the brittle inclusion on the material ductility and are consistent with experimental observations [31].

\subsubsection{Macroscopic energies}

The ability of the proposed numerical periodic homogenization approach is here illustrated by the macroscopic influence of fraction of inclusions on the energy balance during the fracture process. The considered energies are the elastic energy, the plastic energy and the surface energy. This surface energy is composed by the stored energy and the dissipated energy along the cohesive zones during the fracture process.

Fig. 17 shows the ratio of the energies (elastic, plastic and surface) to the total energy (sum of the considered energies) vs the ratio of time to final time for $30 \%$ hydrided Zircaloy- 4 . Before the onset of fracture, the contri-

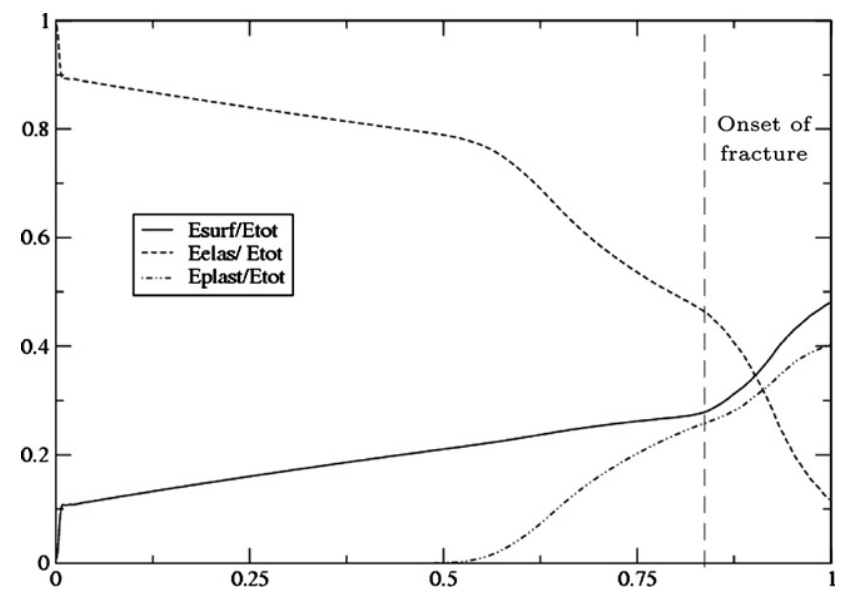

Fig. 17. Ratio of elastic energy $E_{\text {elas }}$, plastic energy $E_{\text {plast }}$ and surface energy $E_{\text {surf }}$ (dissipated + stored along the cohesive zones) to total energy $E_{\text {tot }}$ (elastic + plastic + surface) vs ratio of time to final time for $30 \%$ hydrided Zircaloy- 4 .

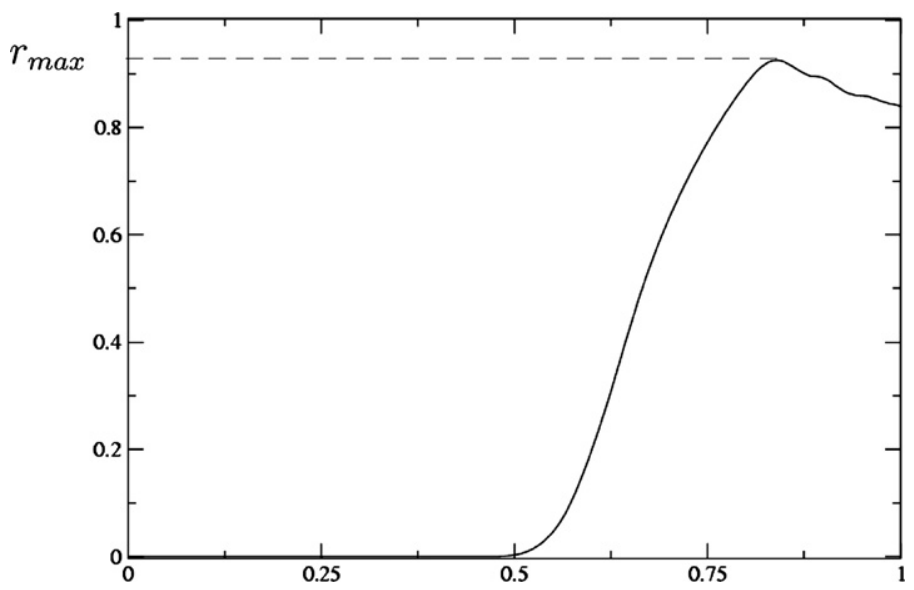

Fig. 18. Ratio of plastic energy to cohesive energy vs ratio of time to final time for $30 \%$ hydrided Zircaloy- 4 . 


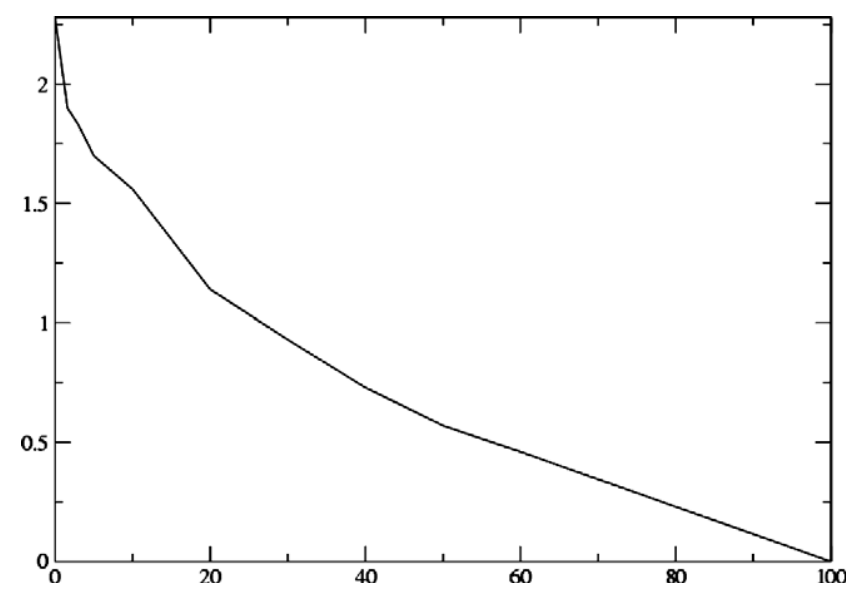

Fig. 19. Maximal ratio of plastic energy to cohesive energy ( $r_{\max }$ in Fig. 18) vs fraction of inclusions (\%).

bution of elastic strain energy decreases in favour of the surface energy (during overall elastic process) and the plastic energy (during overall plastic process). As previously mentioned, when the fracture occurs, the elastic strain energy is released and thus the contribution of this energy decreases rapidly in favour of the surface energy.

The ratio of the plastic energy to cohesive energy vs the ratio of time to final time is plotted in Fig. 18. This ratio increases during the overall plastic process and decreases during the damage process. This phenomenon can be explained by the fact that the surface energy increases rapidly at the onset of fracture even though the increase of the plastic energy remains monotonous. The maximum value reached by the ratio is denoted $r_{\max }$.

Fig. 19 shows the evolution of this parameter as a function of the fraction of inclusions. The value decreases with the fraction to reach zero when the composite corresponds to an elastic material ( $100 \%$ of hydride). This illustrates results from the progressive transition of the composite material from ductile matrix to brittle inclusions with the fraction of inclusions.

\section{Conclusion}

This paper presented a numerical framework, based on micromechanical concept, for the fracture of heterogeneous materials. The micromechanical method consists in a multibody approach and Frictional Cohesive Zone Model in the Non-Smooth Contact Dynamics framework. The effective properties are obtained by periodic numerical homogenization. A two field Finite Element formulation and an extension of the NSCD method was so carried out. The associated software allows to simulate, in finite deformation, from crack initiation to post-fracture material in heterogeneous materials. The ability of the framework was illustrated by simulations of a heterogeneous material from the nuclear industry. Particularly, the weakening effect of the hydride inclusions has been emphasised.

\section{Appendix A. Non-standard quantities of the discrete problem (31)}

The non-standard quantities $\boldsymbol{N}, \mathbf{r}, \boldsymbol{G}$ and $\boldsymbol{H}$ of the discrete problem (31) are:

$$
\begin{aligned}
& r_{I}=\sum_{e=1}^{\tau} \int_{\partial K_{0}^{e}} \mathbf{R}\left(\left[\mathbf{q}^{\#}\right]\right) \cdot \boldsymbol{\varphi}_{I} \mathrm{~d} S \quad 0 \leqslant I \leqslant N_{\mathbf{u}}^{\mathrm{dof}} \\
& N_{I J}=\sum_{e=1}^{\tau} \int_{K_{0}^{e}} \rho \boldsymbol{\varphi}_{I} \cdot\left(\boldsymbol{\pi}_{J} \cdot \mathbf{X}\right) \mathrm{d} x \quad 0 \leqslant I \leqslant N_{\mathbf{u}}^{\mathrm{dof}}, 0 \leqslant J \leqslant N_{\overline{\boldsymbol{F}}}^{\mathrm{dof}}, \\
& G_{I}=-\sum_{e=1}^{\tau} \int_{K_{0}^{e}} \boldsymbol{\Pi}\left(\mathbf{q}^{\#}, \overline{\mathbf{d}}\right): \pi_{I} \mathrm{~d} x \quad 0 \leqslant I \leqslant N_{\overline{\boldsymbol{F}}}^{\mathrm{dof}}, \\
& H_{I}=\left|\Omega_{0}\right| \overline{\boldsymbol{\Pi}}: \pi_{I} \quad 0 \leqslant I \leqslant N_{\overline{\boldsymbol{F}}}^{\mathrm{dof}} .
\end{aligned}
$$




\section{Appendix B. Derivation of the dynamic equation and integration over time}

Consider the discrete dynamic system (31). Since unilateral constraints occur, the derivatives are understood in the sense of distributions. The dynamic system is thus written as a measure differential equation:

$$
\begin{aligned}
& \boldsymbol{M}\left(\mathbf{q}^{\#}, t\right) \cdot \mathrm{d} \dot{\mathbf{q}}^{\#}+\boldsymbol{N}\left(\mathbf{q}^{\#}, t\right) \cdot \mathrm{d} \dot{\overline{\mathbf{d}}}=\boldsymbol{F}\left(\mathbf{q}^{\#}, \dot{\mathbf{q}}^{\#}, \overline{\mathbf{d}}, \dot{\overline{\mathbf{d}}}, t\right) \mathrm{d} t+\mathbf{r d} v, \\
& 0=\boldsymbol{G}\left(\mathbf{q}^{\#}, \dot{\mathbf{q}}^{\#}, \overline{\mathbf{d}}, \dot{\overline{\mathbf{d}}}, t\right) \mathrm{d} t+\boldsymbol{H}(t) \mathrm{d} t,
\end{aligned}
$$

where $\mathrm{d} t$ is a Lebesgue measure, $\mathbf{r} \mathrm{d} v$ denote the contact impulsions and $\mathrm{d} v$ is a positive measure.

Denoting by a subscript $i$ quantities at a time $t_{i}$ and by $i+1$ quantities at time $t_{i+1}$, (B.1a) and (B.1b) are rewritten as follows:

$$
\left\{\begin{array}{l}
\int_{t_{i}}^{t_{i+1}} \boldsymbol{M}\left(\mathbf{q}^{\#}, t\right) \cdot \mathrm{d} \dot{\mathbf{q}}^{\#}+\int_{t_{i}}^{t_{i+1}} \boldsymbol{N}\left(\mathbf{q}^{\#}, t\right) \cdot \mathrm{d} \dot{\overline{\mathbf{d}}} \\
\quad=\int_{t_{i}}^{t_{i+1}} \boldsymbol{F}\left(\mathbf{q}^{\#}, \dot{\mathbf{q}}^{\#}, \overline{\mathbf{d}}, \dot{\overline{\mathbf{d}}}, t\right) \mathrm{d} t+\int_{] t_{i}, t_{i+1}\right]} \mathbf{r} \mathrm{d} v \\
0=\int_{t_{i}}^{t_{i+1}} \boldsymbol{G}\left(\mathbf{q}^{\#}, \dot{\mathbf{q}}^{\#}, \overline{\mathbf{d}}, \dot{\overline{\mathbf{d}}}, t\right) d t+\int_{t_{i}}^{t_{i+1}} \boldsymbol{H}(t) \mathrm{d} t \\
\mathbf{q}\left(t_{i+1}\right)=\mathbf{q}\left(t_{i}\right)+\int_{t_{i}}^{t_{i+1}} \dot{\mathbf{q}}(\tau) \mathrm{d} \tau \\
\overline{\mathbf{d}}\left(t_{i+1}\right)=\overline{\mathbf{d}}\left(t_{i}\right)+\int_{t_{i}}^{t_{i+1}} \dot{\overline{\mathbf{d}}}(\tau) \mathrm{d} \tau
\end{array}\right.
$$

\section{Appendix C. Newton-Raphson algorithm}

The nonlinear system (32) is solved using a Newton-Raphson algorithm. The problem consists in finding the minimal root of the residues:

$$
\left\{\begin{array}{l}
\mathscr{F}\left(\dot{\mathbf{q}}_{i+1}^{\#}, \dot{\overline{\mathbf{d}}}_{i+1}, \mathbf{r}_{i+1}\right):=\boldsymbol{M}\left(\mathbf{q}_{i+1}^{\#}, t_{i+1}\right) \cdot\left(\dot{\mathbf{q}}_{i+1}^{\#}-\dot{\mathbf{q}}_{i}^{\#}\right) \\
\quad+\boldsymbol{N}\left(\mathbf{q}_{i+1}^{\#}, t_{i+1}\right) \cdot\left(\dot{\overline{\mathbf{d}}}_{i+1}-\dot{\overline{\mathbf{d}}}_{i}\right)-h_{i}\left\{(1-\theta) \boldsymbol{F}_{i}+\theta \boldsymbol{F}_{i+1}\right\}-h_{i} \mathbf{r}_{i+1}, \\
\mathscr{G}\left(\dot{\mathbf{q}}_{i+1}^{\#}, \dot{\overline{\mathbf{d}}}_{i+1}\right):=-h_{i}\left\{(1-\theta) \boldsymbol{G}_{i}+\theta \boldsymbol{G}_{i+1}\right\}-h_{i}\left\{(1-\theta) \boldsymbol{H}_{i}+\theta \boldsymbol{H}_{i+1}\right\} .
\end{array}\right.
$$

Particularly, at the iteration $k+1$, the linear system to solve is:

$$
\mathbf{J}\left(\left(\dot{\mathbf{q}}_{i+1}^{\#}\right)^{k}, \dot{\overline{\mathbf{d}}}_{i+1}^{k}, \mathbf{r}_{i+1}^{k}\right) \cdot\left\{\begin{array}{c}
\left(\dot{\mathbf{q}}_{i+1}^{\#}\right)^{k+1}-\left(\dot{\mathbf{q}}_{i+1}^{\#}\right)^{k} \\
\dot{\overline{\mathbf{d}}}_{i+1}^{k+1}-\dot{\overline{\mathbf{d}}}_{i+1}^{k} \\
\mathbf{r}_{i+1}^{k+1}-\mathbf{r}_{i+1}^{k}
\end{array}\right\}=-\left\{\begin{array}{c}
\mathscr{F}\left(\left(\dot{\mathbf{q}}_{i+1}^{\#}\right)^{k}, \dot{\overline{\mathbf{d}}}_{i+1}^{k}, \mathbf{r}_{i+1}^{k}\right) \\
\mathscr{G}\left(\left(\dot{\mathbf{q}}_{i+1}^{\#}\right)^{k}, \dot{\overline{\mathbf{d}}}_{i+1}^{k}\right)
\end{array}\right\}
$$

where, using the notations $\mathscr{F}:=\mathscr{F}\left(\dot{\mathbf{q}}^{\#}, \dot{\overline{\mathbf{d}}}, \mathbf{r}\right)$ and $\mathscr{G}:=\mathscr{G}\left(\dot{\mathbf{q}}^{\#}, \dot{\overline{\mathbf{d}}}, \mathbf{r}\right)$, the Jacobian matrix, denoted $\mathbf{J}$, is defined by:

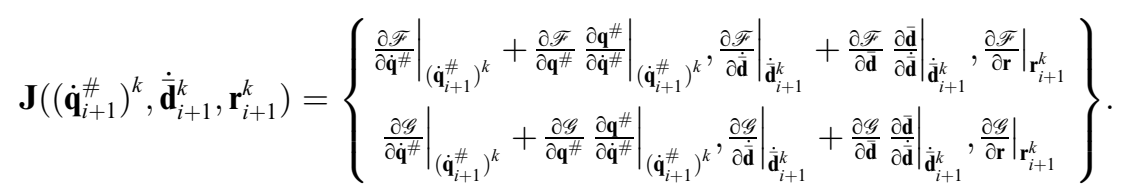

Derivating Eqs. (33) and using the slow variation of $\boldsymbol{M}\left(\mathbf{q}^{\#}, t\right)$ and $\boldsymbol{N}\left(\mathbf{q}^{\#}, t\right)$ with $\mathbf{q}^{\#}$, the Jacobian (C.1) can be rewritten:

$$
\mathbf{J}\left(\left(\dot{\mathbf{q}}_{i+1}^{\#}\right)^{k}, \dot{\overline{\mathbf{d}}}_{i+1}^{k}, \mathbf{r}_{i+1}^{k}\right)=\left\{\begin{array}{l}
\left(\mathbf{w}_{\mathbf{q}^{\#} \mathbf{q}^{\#}}^{k}\right)^{-1},\left(\mathbf{w}_{\mathbf{q}^{\#} \dot{\overline{\mathbf{d}}}}^{k}\right)^{-1},-h_{i} \\
\left(\mathbf{w}_{\dot{\mathbf{d}} \mathbf{q}^{\#}}^{k}\right)^{-1},\left(\mathbf{w}_{\dot{\mathbf{d}} \boldsymbol{\dot { d }}}^{k}\right)^{-1}, 0
\end{array}\right\},
$$

where $\boldsymbol{F}$ and $\boldsymbol{G}$ denote respectively $\boldsymbol{F}\left(\mathbf{q}^{\#}, \dot{\mathbf{q}}^{\#}, \overline{\mathbf{d}}, \dot{\overline{\mathbf{d}}}, t\right)$ and $\boldsymbol{G}\left(\mathbf{q}^{\#}, \dot{\mathbf{q}}^{\#}, \overline{\mathbf{d}}, \dot{\overline{\mathbf{d}}}, t\right)$ and: 


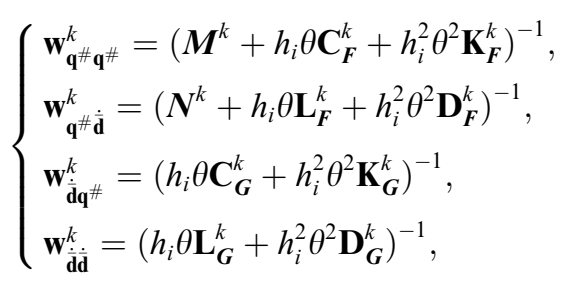

with

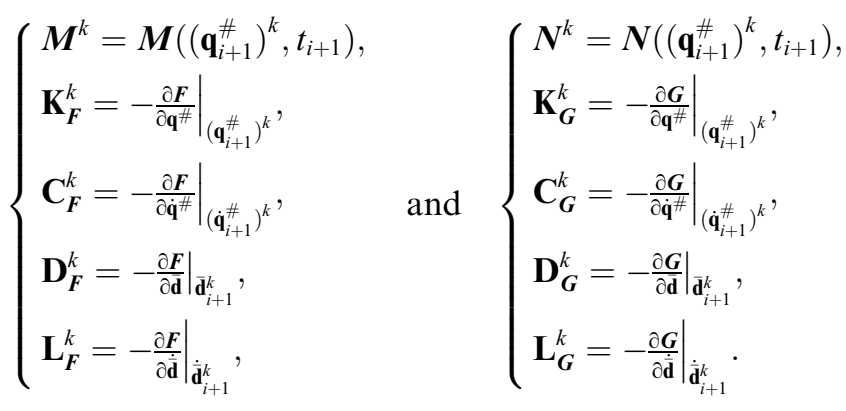

\section{References}

[1] Cox BN, Gao D, Gross H, Rittel D. Modern topics and challenges in dynamic fracture. J Mech Phys Solids 2005;53:565-96.

[2] Perales F, Monerie Y, Dubois F, Stainier L. Numerical simulation of dynamical fracture in heterogeneous materials. In: Bathe K, editor. Computational fluid and solid mechanics. Elsevier, Massachusetts Institute of Technology; 2005. p. 437-41.

[3] Perales F, Monerie Y, Dubois F, Stainier L. Computational non-smooth fracture dynamics in nonlinear and heterogeneous materials. In: Zhou Y, Yu S, Xu Y, editors. Application to fracture of hydrided Zircaloy. Structural mechanics in reactor technology, vol. 18. Beijing, China: Atomic Energy Press; 2005. p. 274-80.

[4] Raous M, Cangémi L, Cocu M. A consistent model coupling adhesion, friction and unilateral contact. Comput Methods Appl Mech Engng 1999;177:383-99.

[5] Raous M, Monerie Y. Unilateral contact, friction and adhesion: 3D crack in composite material. In: Martins J, Monteiro Marques M, editors. 3d contact mechanics international symposium, collection solid mechanics and its applications. Peniche (Portugal): Kluwer; 2001. p. 333-46.

[6] Needleman A. A continuum model for void nucleation by inclusion debonding. J Appl Mech 1987;54:525-31.

[7] Tvergaard V, Hutchinson JW. The relation between crack growth resistance and fracture process parameters in elastic-plastic solids. J Mech Phys Solids 1992;40:1377-97.

[8] Monerie Y, Acary V. Formulation dynamique d'un modèle de zone cohésive tridimensionnel couplant endommagement et frottement. Revue Européenne des Elements Finis 2001;10:489-503.

[9] Moreau J-J. Unilateral contact and dry friction in finite freedom dynamics. In: Moreau J-J, Panagiotopoulos P, editors. Non-smooth mechanics and applications. CISM - courses and lectures, vol. 302. Vienna: Springer; 1988. p. 1-82.

[10] Jean M. The non-smooth contact dynamics method. Comput Methods Appl Mech Engng 1999;177:235-57.

[11] Michel J-C, Moulinec H, Suquet P. Effective properties of composite materials with periodic microstructure: a computational approach. Comput Methods Appl Mech Engng 1999;172:109-43.

[12] Kanit T, Forest S, Galliet I, Mounoury V, Jeulin D. Determination of the size of the representative volume element for random composites: statistical and numerical approach. Int J Solids Struct 2003;40:3647-79.

[13] Dubois F, Jean M. Une plateforme de développement dédiée la modélisation des problèmes d'interaction. In: Potier-Ferry M, Bonnet M, Bignonnet A, editors. 6ème colloque national en calcul de structures, (vol. 1). Giens; 2003. p. 111-8.

[14] Piar B, Chailan L, Vola D. Scientific software architectures designed to survive change. In: Trend in physical and numerical of multiphase industrial flows. Cargèse, 2003.

[15] Stainier L, Dubois F, Peyroux R. MatLib, une bibliothèque portable de modèles constitutifs pour la mécanique non-linéaire des solides: concepts et implémentation. In: Potier-Ferry M, Bonnet M, Bignonnet A, editors. Gème Colloque National en Calcul des Structures, (vol. 3). Giens; 2003. p. 25-32.

[16] Fremond M. Adhérence des solides. J Méc Théor Appl 1987;6:383-407.

[17] Jean M, Acary V, Monerie Y. Non-smooth contact dynamics approach of cohesive materials. Phil Trans R Soc Lond A 2001;359:2497-518.

[18] Sanchez-Palencia E. Comportement local et macroscopique d'un type de milieux physiques heterogenes. Int $\mathbf{J}$ Engng Sci 1974;12:331-51.

[19] Moulinec H, Suquet P. A numerical method for computing the overall response of nonlinear composites with complex microstructure. Comput Methods Appl Mech Engng 1998;157:69-94. 
[20] Miehe C, Schotte J, Schröder J. Computational micro-macro transitions and overall moduli in the analysis of polycrystals at large strains. Comput Mater Sci 1999;16:372-82.

[21] Miehe C, Schröder J, Schotte J. Computational homogenization analysis in finite plasticity of texture development in polycrystalline materials. Comput Methods Appl Mech Engng 1999;171:387-418.

[22] Léné F. Contribution à l'étude des matériaux composites et de leur endommagement. PhD thesis, Université de Paris VI (1984).

[23] Chaboche J, Feyel F, Monerie Y. Interface debonding model: a viscous regularization with a limited rate dependency. Int J Solids Struct 2001;38(18):3127-60.

[24] Perales F, Monerie Y, Dubois F, Stainier L. Simulation numérique de la rupture dynamique des matériaux hétérogènes. In: Ohayon ARR, Grellier J-P, editors. septième colloque national en Calcul des structures, (vol. 2). Hermes, Giens; 2005. p. 463-8.

[25] Balourdet M, Bernaudat C, Basini V, Hourdequin N. The PROMETRA program: assessment of mechanical properties of Zircaloy-4 fuel properties during a RIA. In: Transactions of the 15 th international conference on structural mechanics in reactor technology, Seoul, Korea, 1999. p. 485-92.

[26] Cazalis B, Bernaudat C, Yvon P, Desquines J, Poussard C, Averty X. The PROMETRA program: a reliable material database for highly irradiated Zircaloy-4, Zirlo ${ }^{\mathrm{TM}}$ and $\mathrm{M} 5^{\mathrm{TM}}$ fuel claddings. In: 18th international conference on structural mechanics in reactor technology, Beijing, China, 2005. p. 383-93, SMiRT18 - C02_1.

[27] Yamanaka S, Yoshioka K, Uno M, Katsura M, Anada H, Matsuda T, et al. Thermal and mechanical properties of zirconium hydride. J Alloys Compd 1999;293-295:23-9.

[28] Yamanaka S, Yamada K, Kurosaki K, Uno M, Takeda K, Anada H, et al. Thermal properties of zirconium hydride. J Nucl Mater 2001;294:94-8.

[29] Acary V, Monerie Y. Nonsmooth fracture dynamics using a cohesive zone approach. Research Report 6032, INRIA, 2006. p. 111-8.

[30] Tijssens M, Sluys L, van der Giessen E. Numerical simulation of quasi-brittle fracture using damaging cohesive surface. Eur J Mech A/Solids 2000;19:761-79.

[31] Garde AM, Smith GP, Pirek RC. Effects of hydride precipitate localization and neutron fluence on the ductility of irradiated Zircaloy-4. In: Bradley ER, Sabol GP, editors. Zirconium in the nuclear industry: eleventh international symposium, ASTM STP 1295. American Society for Testing and Materials; 1996. p. 407-30. 